\title{
On homogeneous ice formation in liquid clouds
}

\author{
B. Kärcher ${ }^{\mathrm{a} \star}$ and A. Seifert ${ }^{\mathrm{b}}$ \\ ${ }^{a}$ German Aerospace Agency (DLR), Institute for Atmospheric Physics (IPA), Wessling, Germany \\ ${ }^{\mathrm{b}}$ Department for Research and Development, German Weather Service (DWD), Offenbach, Germany
}

*Correspondence to: B. Kärcher, DLR Oberpfaffenhofen, IPA, 82234 Wessling, Germany. E-mail: bernd.kaercher@dlr.de

\begin{abstract}
Primary ice formation by homogeneous freezing of supercooled cloud droplets in moist adiabatic air parcels is investigated theoretically and by means of numerical simulations. Dependencies of nucleated ice crystal number and size and associated freezing temperatures are systematically studied as functions of updraught speed and cloud droplet number and size. Droplet freezing temperatures range between 235 and $239 \mathrm{~K}$, depending on dynamical forcing and liquid water content. Vertical resolutions on the order of $10 \mathrm{~m}$ are required in numerical cloud models to resolve the thin layers in which homogeneous ice formation processes unfold. Only a fraction of droplets freeze in weakly forced clouds. Small-scale variability in updraught speeds encountered by air parcels containing supercooled cloud droplets results in broad ice crystal number distributions when sampled across a parcel ensemble. The strong dynamical control of ice initiation in tropospheric clouds initiates homogeneous freezing in the presence of heterogeneous ice nuclei even at moderate mean updraught speeds, suggesting that such particles play a limited role in affecting droplet freezing in cold convective cloud outflow regions. These findings have important implications for microphysical and optical properties of condensed-phase convective detrainment, setting the stage for the evolution of anvil cirrus.
\end{abstract}

Key Words: cloud physics; glaciation; homogeneous freezing; convective outflow

Received 7 April 2015; Revised 21 December 2015; Accepted 5 January 2016; Published online in Wiley Online Library 2 March 2016

\section{Introduction}

It is important to know the phase and physical properties of aerosol and cloud particles at the top of mid-level and high tropospheric clouds - anvils and the stratiform cirrus that originate from them - in order to better estimate their effects on the energy balance, the atmospheric moisture budget and regional circulation patterns. Better understanding of the water phase partitioning within frontal and convective mixed-phase clouds bolsters confidence in the ability to simulate their effects on the hydrological cycle, to improve numerical weather prediction models and to better assess their role in cloud-climate feedbacks (Bony et al., 2015). It is of vital importance to untangle interactions between cloud-nucleating aerosol particles and their dynamical environment across a wide range of scales in order to provide more reliable estimates of cloud effects in weather prediction and climate studies (Stevens and Feingold, 2009). Two types of cloud systems frequently associated with the ice phase exemplify these issues: (sub)tropical deep convective circulation systems linking cumulus clouds with high cirrus (Mauritsen and Stevens, 2015) and the wide cloud bands in extratropical cyclones connecting the boundary layer with the upper troposphere (Joos and Wernli, 2012). Both cloud systems exhibit complex interactions between small-scale microphysical processes and the meso- and large-scale flow, with diabatic effects related to water phase and radiative flux changes featuring prominently in their evolution.
Liquid-phase clouds containing water droplets inhabit the troposphere at a wide range of temperature. At temperatures below the freezing point, these clouds are thermodynamically unstable and may transform into mixed-phase and - given enough time - into pure ice clouds (glaciation). For supercooled water clouds to glaciate, the stage in which the first (primary) ice crystals form is crucial, because their number and size affect subsequent cloud development. An increasing ice content in mixed-phase clouds changes their radiative response, leading to decreased net cooling and changes in the precipitation efficiency. At temperatures $T<235 \mathrm{~K}$ - the domain of cold cirrus clouds - liquid cloud droplets can no longer exist due to spontaneous crystallisation of supercooled water (homogeneous freezing).

As pure cloud water droplets do not freeze homogeneously at $T>240 \mathrm{~K}$ within the lifetime of liquid clouds, more efficient ice-nucleating particles (heterogeneous ice nuclei, IN) may catalyse ice formation. A number of IN-induced freezing pathways are known or have been postulated, but much research remains to better understand IN-mediated nucleation modes (Cantrell and Heymsfield, 2005). However, IN number concentrations are much lower $\left(<0.1 \mathrm{~cm}^{-3}\right.$ in background conditions) than those of cloud condensation nuclei $\left(>100 \mathrm{~cm}^{-3}\right)$ or water droplets deriving from them, and are highly variable in the troposphere depending on location and season (DeMott et al., 2010).

Therefore, detailed numerical and analytical studies of supercooled cloud glaciation induced by homogeneous freezing are warranted. The former allow us (in conjunction with 
measurements) to test the basic scientific understanding encompassing all ice nucleation modes and the full suite of dynamical-microphysical interactions, while the latter serve as an aid in this process. The present study extends previous research into homogeneous freezing of supercooled aerosol particles in cold cirrus (Kärcher and Lohmann, 2002) to the mixed-phase regime.

Here we formulate and apply an approximate analytical model for primary homogeneous ice formation in moist adiabatic air parcels containing supercooled liquid water droplets at $T>230 \mathrm{~K}$. We seek an approach to this problem based on cloud theory. We do not attempt to provide an accurate parametrization of this ice formation mode. The conceptual framework is described in section 2, and the mathematical model is developed in section 3. Key results of the theory are discussed in section 4 Comparisons with results from numerical simulations using a cloud parcel model are presented in section 5 . We highlight the crucial role of dynamical forcing in the ice formation process and make a first attempt to assess the role of IN in primary ice formation in section 6. We conclude our study in section 7 .

Notation and acronyms are listed in Appendix D.

\section{Prerequisites}

\subsection{Freezing-relaxation concept}

We develop a conceptual model for the purpose of estimating frozen cloud droplet numbers and sizes in cloud updraught regions. The dynamics of the three-phase water system comprised of water vapour, supercooled liquid water droplets and ice crystals is rather complex, in particular for variable updraught histories (Korolev and Isaac, 2003; Korolev and Field, 2008). Therefore, we idealise the system in order to gain insight into the basic mechanisms at work and to identify key parameters and their mutual dependencies.

A schematic of the evolution of supersaturation over supercooled liquid water, $s_{\mathrm{W}}-$ a measure for condensable water vapour - in a cloud containing water droplets taken from numerical simulations (section 5), is shown in Figure 1 (solid curves). The rate of cooling within a rising cloud parcel is dictated by a constant updraught speed, $w>0$, and thermodynamic properties of the moist adiabat, in particular the lapse rate. Condensational growth of the cloud droplets continuously removes $s_{\mathrm{w}}$ generated by the cooling. Droplets freeze homogeneously into ice crystals governed by a formation rate, $j$,

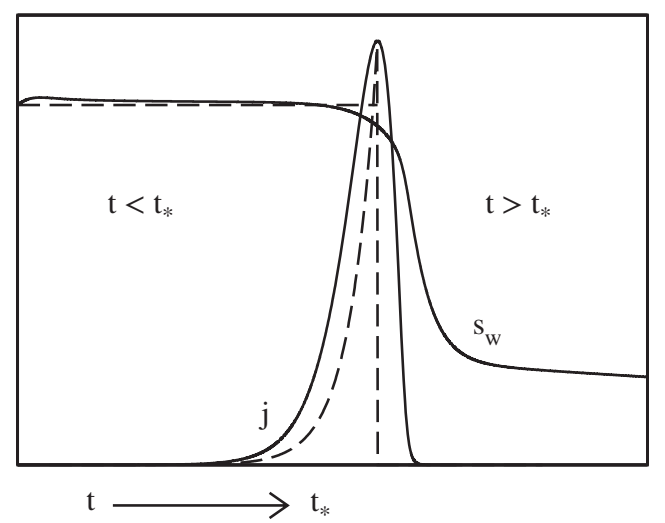

Figure 1. Key variables involved in the ice formation process by homogeneous cloud droplet freezing in an air parcel subject to a constant updraught speed (cooling rate). Shown are the temporal evolution of the ice crystal formation rate due to freezing, $j=\mathrm{d} n_{\mathrm{i}} / \mathrm{d} t$, and of the liquid water supersaturation, $s_{w}$, taken from cloud parcel simulations (solid curves). The subscript $\star$ marks conditions a freezing-relaxation, i.e. the point in time $\left(t=t_{\star}\right)$, where $j$ reaches its peak value. At times $t<t_{\star}$, the presence of cloud droplets ensures that $s_{w}$ quickly attains quasi-steady-state value close to saturation, except near $t_{\star}$. The corresponding dashed curves indicate the idealised behaviour of our theoretical approach, where $s_{\mathrm{W}}$ is kept constant and $j$ is approximated by an exponential pulse. The short time period around $t_{\star}$ in which $s_{\mathrm{w}}$ declines and $j$ maximises according to the simulations is replaced by step changes. which increases very rapidly as the temperature decreases. While $j$ reaches its peak, $s_{\mathrm{w}}$ declines from approximately water saturation $\left(s_{\mathrm{W}} \approx 0\right)$ towards a subsaturated state $\left(s_{\mathrm{W}}<0\right)$, wherein yet unfrozen droplets evaporate and might still freeze as long as $j>0$.

We conceptually divide the ice formation process occurring in this three-phase system into an early stage $\left(t<t_{\star}\right)$, wherein the basic physical processes are governed by only two phases, vapour and liquid, and a late stage $\left(t>t_{\star}\right)$, again governed by only two phases, vapour and ice. Both two-phase systems are coupled at the well-defined point in time, $t=t_{\star}$ (hereinafter referred to as freezing-relaxation), at which $j$ takes its maximum value. Prior to $t_{\star}$, we assume that depositional growth of early nucleated ice crystals has not yet affected $s_{W}$. At $t_{\star}$, enough ice crystals have formed so that quenching of $s_{\mathrm{W}}$ sets in abruptly. The freezing process and the associated effect on $s_{\mathrm{w}}$ is described using information available from the contiguous two-phase systems. After $t_{\star}$, the evolution of $j$ and $s_{\mathrm{w}}$ depends on the updraught history, but no longer affects the freezing process. This idealised behaviour, also depicted in Figure 1 (dashed curves), guides the theoretical analysis enabling us to derive a lower-limit estimate for the number of frozen droplets. The fact that the finite time window in which $s_{\mathrm{w}}$ declines collapses into a single instant coinciding with the peak ice formation rate makes this task analytically tractable, but also calls for benchmark numerical simulations for validation.

Regarding the early cloud droplet growth stage, after an equilibration period a quasi-steady state develops due to a competition between sustained cooling increasing $s_{\mathrm{w}}$ and droplet condensational growth decreasing $s_{\mathrm{w}}$. For typical total droplet numbers and mean sizes, the resulting equilibrium supersaturation over liquid water is small and the equilibration time-scale is short (Korolev and Mazin, 2003). In the freezing-relaxation concept, the late ice phase supersaturation relaxation stage is regarded as a consequence of the processes taking place up to $t_{\star}-$ it is therefore not necessary to include this stage in the theoretical model. In reality, a competition between droplet evaporation and freezing occurs in that stage, which ultimately determines the total number of frozen droplets. We make use of numerical simulations in order to explore how this simplification affects the theoretical results.

To further simplify the theoretical analysis, we describe cloud droplets and ice crystals by monodisperse particle populations. We assume that mean radii of cloud droplets are sufficiently small so that collision-coalescence is not initiated and rain drops do not form. Furthermore, a constant updraught speed allows us to elaborate two distinct dynamical regimes within which ice formation events unfold. We neglect ice crystal sedimentation, as we study the onset of ice formation on short time-scales; the underlying assumption that they do not fall out of the homogeneous ice formation region within the time of freezing and initial growth will be assessed later in this study. While we briefly address effects of variability in updraught speeds encountered by an ensemble of air parcels, investigating possible effects of microscale turbulent mixing on ice nucleation is outside the scope of this work.

The first ice crystals experience the highest supersaturation and therefore grow most rapidly. Depending on how fast they are capable of reducing the supersaturation during the freezing event, yet unfrozen droplets may subsequently turn into ice or evaporate. We already see two dynamical regimes of cloud forcing with distinct microphysical signatures emerging. If the dynamical forcing is sufficiently strong (i.e. the updraught speed or the cooling rate is very high), supersaturation is quenched very rapidly and most of the droplets freeze in a burst without significant ice crystal growth, creating a narrow size distribution of small ice crystals. This regime is realised in jet aircraft contrails (Kärcher et al., 2015) or, as shown here, approached in deep convective clouds. Alternatively, most of the droplets may evaporate before freezing is completed when updraught speeds are small, resulting in smaller ice crystal formation rates, slower supersaturation quenching and broader ice crystal size distributions with larger mean sizes. 
We provide quantitative results predicting nucleated ice crystal number concentrations and freezing temperatures at $t_{\star}$ for various updraught speeds and cloud droplet parameters. Mean ice crystal radii and ice water content are also provided. The consequences of the simplifications inherent to our idealised approach are investigated with the help of a newly developed spectral cloud parcel model simulating the full suite of physical interactions in the three-phase water system including homogeneous droplet freezing. This includes the issue of broadening of ice crystal size distributions during and shortly after freezing as an inferred mechanism.

\subsection{Ice supersaturation equation}

We define $S_{\mathrm{i}}$ as the ice saturation ratio between water vapour partial pressure and water saturation vapour pressure over ice. As $s_{\mathrm{W}}$ declines (as shown in Figure 1), the corresponding supersaturation with respect to the ice phase, $s_{\mathrm{i}}=S_{\mathrm{i}}-1$, relaxes from near water saturation $\left(s_{\mathrm{i}} \simeq s_{\mathrm{wi}} \approx 0.4\right)$ towards ice saturation $\left(s_{\mathrm{i}} \rightarrow 0\right)$. We feature the ice supersaturation equation

$$
\frac{\mathrm{d} s_{\mathrm{i}}}{\mathrm{d} t}=\underbrace{a_{1} \cdot w}_{\mathcal{P}}-\underbrace{a_{2} \cdot \mathcal{R}}_{\mathcal{L}}
$$

centre stage in the mathematical development of our theory. In Eq. (1), derived in Appendix $\mathrm{A}, a_{1}$ and $a_{2}$ are coefficients describing physical characteristics of the air parcel ascending in the water-saturated environment at the instant $t_{\star}$. Moreover, $\mathcal{R}$ integrates vapour deposition on simultaneously nucleating and growing ice crystals, representing the removal rate of water molecules from the gas phase, $\mathcal{P}$ is the overall source term for $s_{\mathrm{i}}$ and $\mathcal{L}$ is the overall sink term for $s_{\mathrm{i}}$. The total number concentration, $n_{\mathrm{d}}$, and volume-mean radius, $r_{r m d}$, of the droplet population are used as fixed parameters. They result from the activation of cloud condensation nuclei into water droplets and the condensational growth of cloud droplets prior to $t_{\star}$. The temporal rate of change of supersaturation, $\mathcal{P}(w)$, is constrained by the thermodynamics of the vapour-liquid phase system and increases in proportion to the updraught speed (section 3.1). The microphysical sink term carries information on the characteristics of the ice nucleation and growth mechanisms and the forcing, $\mathcal{R}(w)$ (section 3.3).

In Eq. (1) $w$ represents the vertical component of the smallscale velocity field acting on the rising air parcel evolving along prescribed vertical profiles of air temperature and pressure. We postulate that all particles inside the parcel are subject to the same local environment, preventing them from separating in the turbulent velocity field and thereby experiencing different supersaturation histories. We focus on processes in the interior of a cloud-nucleating pocket of air, so that we can ignore entrainment of drier air at its boundaries, which would tend to slow the forcing of $s_{\mathrm{i}}$.

In reiterating the 'freezing-relaxation' concept, we note that besides depending on $w$ and the water vapour content, $\mathcal{P}$ depends only on droplet variables while $\mathcal{R}$ depends on vapour and ice variables. Both two-phase stages are assumed to evolve completely independently from each other, but are linked together at the instant $t=t_{\star}$, the only point in time where we consider all three water phases together. Correspondingly, we do not integrate Eq. (1) to obtain the time history of ice supersaturation, $s_{\mathrm{i}}(t)$, but seek a solution only at $t_{\star}$ (Figure 1 ) to estimate the number concentration of ice crystals, $n_{\star}=n_{\mathrm{i}}\left(t_{\star}\right)$, sufficient to quench $s_{\mathrm{i}}$ (section 3.4). Hereby, $t_{\star}$ and hence the maximum of $j$ is defined by the assumption that the cloudy parcel is on the one hand in a quasi-steady-state with respect to the liquid phase wherein $s_{\mathrm{w}}$ is unaffected by the presence of early freezing droplets, and on the other hand the quenching of $s_{\mathrm{w}}$ is solely controlled by the ice phase which in turn fully develops at $t_{\star}$.

We note that $n_{\star}$ is a lower bound to the total ice number, as ice crystals may continue to form at $t>t_{\star}$ contrary to our assumption, depending on the updraught history and on how rapidly the nucleation pulse is cut-off by droplet evaporation and freezing. The latter contribution to the total ice crystal number is often small (section 5).

\section{Model development}

\subsection{Supersaturation forcing}

For an air parcel cooling along the moist adiabat, the coefficients in Eq. (1) in the droplet-controlled regime read (Appendix A):

$$
\begin{aligned}
& a_{1}=\frac{S_{\mathrm{wi}}}{T}\left\{\Gamma_{\mathrm{a}}\left(\theta_{\mathrm{w}}-\gamma\right)+\Gamma_{\mathrm{ws}} \theta_{\mathrm{f}}\right\}, \\
& a_{2}=\frac{1}{n_{\mathrm{is}}}\left(1+\mu_{\mathrm{is}} \frac{\theta_{\mathrm{w}} \theta_{\mathrm{i}}}{\epsilon \gamma}\right),
\end{aligned}
$$

where we have introduced the abbreviations

$$
\theta_{\mathrm{w} / \mathrm{i} / \mathrm{f}}=\frac{L_{\mathrm{w} / \mathrm{i} / \mathrm{f}}}{R_{\mathrm{w}} T}, \quad \gamma=\frac{c_{p}}{R_{\mathrm{a}}}, \quad \Gamma_{\mathrm{a}}=\frac{g}{c_{p}}, \quad \mu_{\mathrm{is}}=\epsilon \frac{n_{\mathrm{is}}}{n_{\mathrm{a}}},
$$

and where $\gamma=3.5$ is a coefficient accounting for adiabatic change, $\Gamma_{\mathrm{a}}=9.8 \mathrm{~K} \mathrm{~km}^{-1}$ is the dry adiabatic lapse rate, $\Gamma_{\mathrm{ws}}(p, T)$ is the liquid adiabatic lapse rate, $T$ is the parcel temperature and $p$ is the pressure of dry air in the parcel; $L_{\mathrm{W} / \mathrm{i}}(T)$ is the latent heat of evaporation/sublimation, $L_{\mathrm{f}}(T)=L_{\mathrm{i}}-L_{\mathrm{w}}$ is the latent heat of fusion $\left(\theta_{\mathrm{f}}=\theta_{\mathrm{i}}-\theta_{\mathrm{w}}\right), n_{\mathrm{is}}(T)$ is the number concentration of water molecules in the vapour at ice saturation and $n_{\mathrm{a}}(p, T)$ is the number concentration of air molecules. Furthermore, we introduce the ratio of the mass of molecules of water and dry air, $\epsilon=0.622$, the acceleration of gravity, $g=9.81 \mathrm{~m} \mathrm{~s}^{-2}$, the isobaric specific heat capacity of dry air, $c_{p}=1004.67 \mathrm{~J} \mathrm{~kg}^{-1} \mathrm{~K}^{-1}$ and the specific gas constants for dry air and water vapour, $R_{\mathrm{a}}=287.04 \mathrm{~J} \mathrm{~kg}^{-1} \mathrm{~K}^{-1}$ and $R_{\mathrm{w}}=461.4 \mathrm{~J} \mathrm{~kg}^{-1} \mathrm{~K}^{-1}$, respectively. Below $240 \mathrm{~K}$, the second terms in brackets in Eqs (2) and (3) are small, i.e. $a_{1} \simeq S_{\mathrm{wi}} \Gamma_{\mathrm{a}}\left(\theta_{\mathrm{w}}-\gamma\right) / T$ and $a_{2} \simeq 1 / n_{\mathrm{is}}$.

In the vapour-liquid system, thermodynamic equilibrium - embodied in the use of $S_{\text {wi }}$ in Eq. (2) - is not strictly established in the presence of dynamical forcing. However, since associated peak supersaturations stay small $\left(s_{\mathrm{W}} \ll 0.1\right)$ at typical droplet concentrations and sizes and for updraught speeds $<10 \mathrm{~m} \mathrm{~s}^{-1}$ (Korolev and Mazin, 2003), the assumption of liquid water saturation in the droplet growth stage is quite accurate.

Cooling rates, $\mathrm{d} T / \mathrm{d} t<0$, in a water-saturated air parcel in the absence of ice are tied to the updraught speed and the saturated (liquid) adiabatic lapse rate, $\Gamma_{\mathrm{s}}$ (Stevens, 2005):

$$
\frac{\mathrm{d} T}{\mathrm{~d} t}=-\underbrace{\Gamma_{\mathrm{a}}\left(\frac{1+\mu_{\mathrm{ws}} \theta_{\mathrm{w}}}{1+\mu_{\mathrm{ws}} \theta_{\mathrm{w}}^{2} / \gamma}\right)}_{\Gamma_{\mathrm{ws}}} \cdot w,
$$

where $\mu_{\mathrm{ws}}(p, T)=\epsilon n_{\mathrm{ws}} / n_{\mathrm{a}}$ is the mass mixing ratio of water molecules in the vapour at liquid water saturation. Deviations between $\Gamma_{\text {ws }}$ from Eq. (5) and the liquid adiabatic lapse rate are small, because the contribution of liquid condensate to the total specific heat capacity is small; $\Gamma_{\mathrm{ws}}$ asymptotes to the dry adiabatic lapse rate, $\Gamma_{\mathrm{a}}$, at $T<240 \mathrm{~K}$.

Taken together, the ice supersaturation production rate in Eq. (1) is given by

$$
\mathcal{P}=a_{1} w=S_{\mathrm{wi}} \frac{\vartheta}{T}\left\{\frac{\Gamma_{\mathrm{a}}}{\Gamma_{\mathrm{ws}}}\left(\theta_{\mathrm{w}}-\gamma\right)+\theta_{\mathrm{f}}\right\},
$$

with the absolute value of the cooling rate, $\vartheta=-\mathrm{d} T / \mathrm{d} t>0$.

\subsection{Ice nucleation and diffusional growth}

The ice crystal formation rate due to nucleation, $j=\mathrm{d} n_{\mathrm{i}} / \mathrm{d} t$, gives the number of ice crystals per unit volume of air formed 
per unit time. A more fundamental quantity is the nucleation rate coefficient, $J$, signifying the number of ice germs nucleating homogeneously per unit volume of supercooled liquid water per unit time. In the case of volume-controlled droplet freezing, $j$ is given by

$$
j=\mathrm{LWV} \cdot J, \quad \mathrm{LWV}=V_{\mathrm{d}} n_{\mathrm{d}}, \quad V_{\mathrm{d}}=\frac{4 \pi}{3} r_{\mathrm{d}}^{3},
$$

where LWV is the liquid water volume fraction (per unit volume of air) of $n_{\mathrm{d}}$ droplets with volume-mean radius, $r_{\mathrm{d}}$, or single-particle volume, $V_{\mathrm{d}}$. Once ice nucleation commences within a droplet, its entire water volume turns into ice without a significant time delay, because crystallisation proceeds extremely rapidly (Matsumoto et al., 2002).

To derive an estimate of $n_{\mathrm{i}}(t)$, the number of ice crystals at time $t$ nucleating between an early time $t^{\prime}$ and $t^{\prime}+\mathrm{d} t^{\prime}$, we approximate the ice crystal formation rate by an exponential pulse (Ford, 1998):

$$
j\left(t, t^{\prime}\right)=j(t) \mathrm{e}^{-\left(t-t^{\prime}\right) / \tau_{\mathrm{n}}}, \quad-\infty<t^{\prime} \leq t, \quad t \leq t_{\star},
$$

with the time-scale, $\tau_{\mathrm{n}}$, measuring the duration of the freezing event. Later, we identify $t$ with the time of freezing-relaxation, $t_{\star}$, where $j$ from Eq. (8) takes its maximum. The assumption of a nucleation pulse is reasonable (Figure 1). The formation rate is close to zero at early times because it is too warm for freezing to take place. It is also close to zero at late times, because all droplets have already frozen or evaporated. The exponential shape allows for a simple analytic solution, but may not accurately track the ice crystal formation rate near its maximum (Figure 1).

Integrating Eq. (8) from the remote past up to time $t$ leads to the nucleated ice crystal number concentration

$$
n_{\mathrm{i}}^{(1)}=\int_{-\infty}^{t \leq t_{\star}} j\left(t, t^{\prime}\right) \mathrm{d} t^{\prime}=j(t) \tau_{\mathrm{n}} \leq n_{\mathrm{d}} .
$$

Equation (9) predicts the number concentration of ice crystals that form from the cloud droplet population in a nucleation pulse increasing exponentially with the time-scale $\tau_{\mathrm{n}}$. We added the superscript (1) to indicate that we seek a second relationship to finally estimate $n_{\star}=n_{\mathrm{i}}\left(t_{\star}\right)$, since $t_{\star}\left(\right.$ or $\left.T_{\star}\right)$ is not known a priori.

We compute $J(T)$ from a parametrization derived from laboratory measurements (Riechers et al., 2013):

$$
\ln J=-\frac{T-235}{\delta T}+19.44
$$

with $J$ in $\mathrm{cm}^{-3} \mathrm{~s}^{-1}$ and the (inverse) slope parameter, $\delta T=0.28 \mathrm{~K}$. The time-scale, $\tau_{\mathrm{n}}$, of the cooling-induced homogeneous freezing event is chosen to match the exponential time dependence of $j$ :

$$
\tau_{\mathrm{n}}^{-1} \equiv \frac{\partial \ln (j)}{\partial t}=\frac{\partial \ln (J)}{\partial t}+\frac{\partial \ln (\mathrm{LWV})}{\partial t}=\frac{\vartheta}{\delta T}(1+K),
$$

as derived in Appendix B. The contribution of LWV changes to $\tau_{\mathrm{n}}$ is embodied in the factor $K$, which is usually small $(K \ll 1)$ except for unusually low LWV, hence $\tau_{\mathrm{n}} \simeq\{\partial \ln (J) / \partial t\}^{-1}=\delta T / \vartheta$ is largely controlled by changes in $J$ due to cooling.

Combining Eqs (9) and (11), the nucleated ice crystal number concentration follows from

$$
n_{\mathrm{i}}^{(1)}=\min \left\{\mathrm{LWVJ} \tau_{\mathrm{n}} ; n_{\mathrm{d}}\right\} .
$$

The molecular mean free path in mid- and upper-tropospheric air is much smaller than typical droplet radii. Therefore, applying the diffusional growth rate is appropriate for ice crystals in such conditions (Pruppacher and Klett, 1997):

$$
\frac{\mathrm{d} r_{\mathrm{i}}}{\mathrm{d} t}=\frac{b_{\mathrm{i}}}{r_{\mathrm{i}}}, \quad b_{\mathrm{i}}=\frac{\nu D n_{\mathrm{is}} s_{\mathrm{i}}}{1+B_{\mathrm{i}}},
$$

with the volume of a water molecule in ice, $v=3 \times 10^{-29} \mathrm{~m}^{3}$, the diffusion coefficient of water molecules in air, $D(p, T)$, and the factor, $B_{\mathrm{i}}(p, T)$, accounting for the effect of latent heat release during growth:

$$
B_{\mathrm{i}}=\frac{m_{\mathrm{w}} n_{\mathrm{is}} D L_{\mathrm{i}}}{k_{\mathrm{a}} T}\left(\theta_{\mathrm{i}}-1\right)
$$

with the molecular mass of water, $m_{\mathrm{w}}=3 \times 10^{-26} \mathrm{~kg} ; k_{\mathrm{a}}(T)$ is the thermal conductivity of air. To evaluate $D$ and $B_{\mathrm{i}}$, we obtain the parcel's air pressure, $p(T)$, from the US Standard Atmosphere, for simplicity. For initial growth, $s_{\mathrm{i}} \simeq s_{\mathrm{wi}}$ (section 2.2). In the diffusion limit, $D$ and $k_{\mathrm{a}}$ are not corrected for imperfect molecular/thermal accommodation at, and ventilation of, the ice crystal surface. While $B_{\mathrm{i}}$ is not affected very much, this tends to slightly overestimate the radial growth rate. For $T<240 \mathrm{~K}$, $b_{\mathrm{i}}<3 \mu \mathrm{m}^{2} \mathrm{~s}^{-1}$ from Eq. (13) leading to $B_{i}<0.1$. We use a volume-equivalent spherical radius, $r_{i}$, for the ice crystals, ignoring particle shape (capacitance) corrections in Eqs (13) and (14), as the ice crystals undergo only a short growth stage. The curvature (Kelvin) effect is omitted since it reduces $s_{\mathrm{i}}$ (and thereby the growth rate) by less than $1 \%$ over the surfaces of $\mu \mathrm{m}$-sized ice crystals.

The time-scale for initial growth is chosen to match the ice crystal areal growth rate:

$$
\tau_{\mathrm{g}}^{-1} \equiv \frac{1}{r_{\mathrm{i}}^{2}} \frac{\mathrm{d} r_{\mathrm{i}}^{2}}{\mathrm{~d} t} \simeq \frac{2 b_{\mathrm{i}}}{r_{\mathrm{d}}^{2}} .
$$

We encapsulate the basic physics of the freezing-relaxation process into the dimensionless parameter

$$
\kappa \equiv \frac{\tau_{\mathrm{n}}}{\tau_{\mathrm{g}}} \simeq \frac{2 b_{\mathrm{i}}}{r_{\mathrm{d}}^{2}} \frac{\delta T}{\vartheta}
$$

used to distinguish different dynamical forcing (growth) regimes; $\kappa$ varies only slightly with $T$ within the range of conceivable homogeneous freezing temperatures (section 4).

We recall from section 2.1 that supersaturation quenching sets in when nucleated ice crystals begin to significantly deplete the water vapour by depositional growth. The fast growth regime is realised when the ice crystal growth time-scale is much shorter than the time over which further ice crystals can form $(\kappa \gg 1)$. In the opposite slow growth regime, the freezing time-scale is much shorter than the time over which freshly nucleated ice crystals increase significantly in size $(\kappa \ll 1)$.

\subsection{Supersaturation sink}

The freezing growth integral Eq. (1), defined as the timedependent total removal rate of water molecules due to deposition onto the nucleating ice crystals, is given by:

$$
\mathcal{R}=\frac{4 \pi}{v} \int_{-\infty}^{t} j r_{\mathrm{i}}^{2} \frac{\mathrm{d} r_{i}}{\mathrm{~d} t} \mathrm{~d} t^{\prime}=\frac{4 \pi b_{\mathrm{i}}}{v} \int_{-\infty}^{t} j\left(t, t^{\prime}\right) r_{i}\left(t, t^{\prime}\right) \mathrm{d} t^{\prime} .
$$

The solution of the diffusional growth law (Eq. (13)) taken at time $t$ (or corresponding temperature $T$ ), later to be identified with $t_{\star}\left(T_{\star}\right)$, reads

$$
r_{\mathrm{i}}\left(t, t^{\prime}\right)=r_{\mathrm{d}}\left\{1+\kappa \frac{\left(t-t^{\prime}\right)}{\tau_{\mathrm{n}}}\right\}^{1 / 2}
$$

Inserting Eqs (8) and (18) into Eq. (17) leads to (Appendix C)

$$
\mathcal{R}=\frac{4 \pi b_{\mathrm{i}}}{v} n_{\mathrm{i}} r_{\mathrm{d}} \underbrace{\left\{1+\frac{\sqrt{\pi \kappa}}{2} \mathcal{F}(\kappa)\right\}}_{\mathcal{G}(\kappa)},
$$


with $\mathcal{F}$ taken from Eq. (C2). Similarly, the mean radius, $r_{\mathrm{i}}$, of the ice crystal population follows from

$$
r_{\mathrm{i}}=\frac{1}{n_{\mathrm{i}}} \int_{-\infty}^{t} j\left(t, t^{\prime}\right) r_{\mathrm{i}}\left(t, t^{\prime}\right) \mathrm{d} t^{\prime}=r_{\mathrm{d}} \mathcal{G}(\kappa) .
$$

The supersaturation sink term can then be cast into the form

$$
\mathcal{L}=a_{2} \mathcal{R}=a_{2} \frac{4 \pi b_{\mathrm{i}}}{v} n_{\mathrm{i}} r_{\mathrm{i}} \simeq 4 \pi D s_{\mathrm{i}} n_{\mathrm{i}} r_{\mathrm{i}}
$$

Equation (21) expresses that ice supersaturation relaxes due to diffusional growth of the freshly nucleated ice crystals (number $n_{\mathrm{i}} \leq n_{\mathrm{d}}$, mean radius $\left.r_{\mathrm{i}} \geq r_{\mathrm{d}}\right)$ on the time-scale $1 /\left(4 \pi D n_{\mathrm{i}} r_{\mathrm{i}}\right)$ and underlines the necessity to model the nucleation pulse and thereby track the simultaneous nucleation and growth of ice crystals in solving Eq. (17).

We define the ice water content as the total mass of cloud ice per unit volume of air: IWC $=\rho_{\mathrm{i}} n_{\mathrm{i}} V_{\mathrm{i}}$, where $\rho_{\mathrm{i}}=972 \mathrm{~kg} \mathrm{~m}^{-3}$ is the bulk mass density of small, compact ice crystals. We discuss the solutions at $t=t_{\star}$ in section 3.4; the physical characteristics of the relaxation stage in the subsequent two-phase vapour-ice system at $t>t_{\star}$, i.e. the temporal evolution $s_{\mathrm{i}}(t)$ following from Eqs (1) and (13) with fixed $n_{\mathrm{i}}$, has been discussed by Korolev and Mazin (2003).

\subsection{Solution at freezing-relaxation}

We recall that we wish to find the value $n_{\star}=n_{\mathrm{i}}\left(t_{\star}\right)$ at freezingrelaxation. Combining Eqs (6) and (21) to satisfy the freezingrelaxation requirement and solving for $n_{\mathrm{i}}$ yields

$$
\mathcal{P} \equiv \mathcal{L}\left(\kappa, n_{i}\right) \quad \Longrightarrow \quad n_{\mathrm{i}}^{(2)}=v \frac{a_{1}}{a_{2}} \frac{w}{4 \pi b_{\mathrm{i}} r_{\mathrm{d}}} \frac{1}{\mathcal{G}(\kappa)},
$$

where we have added the superscript (2) to indicate that this relationship complements $n_{\mathrm{i}}^{(1)}$ from Eq. (12). The effect of the dynamical forcing on $n_{\mathrm{i}}$ appears on the one hand in the supersaturation forcing $\mathcal{P} \propto w$ from Eq. (6), while $\mathcal{G}=r_{\mathrm{i}} / r_{\mathrm{d}}$ encapsulates the microphysical effects of freezing and ice growth as indicated by Eq. (17) on the other. Thus, microphysics impose a further nonlinear $w$-dependence on $n_{\mathrm{i}}$, except for strong forcing. Using the asymptotic scaling behaviour of $\mathcal{G}(\kappa)$ (Eq. (C3)) with $\kappa \propto 1 / w$, we find that, whereas $\mathcal{G} \rightarrow 1$ for slow ice growth (large $w)$ and therefore $n_{\mathrm{i}}^{(2)} \propto w$ increases towards $n_{\mathrm{d}}$, in fast growth conditions (small $w$ ), $\mathcal{G}<1$ and $n_{\mathrm{i}} \propto w^{3 / 2}<n_{\mathrm{d}}$ due to early quenching of supersaturation and enhanced droplet evaporation.

We reiterate that $n_{\mathrm{i}}^{(2)}$ from Eq. (22) results from the self-limiting nature of the nucleation process and predicts the number concentration of frozen droplets required to initiate supersaturation quenching. Alternatively, Eq. (12) predicts the value $n_{\mathrm{i}}^{(1)}$ resulting from an unconstrained nucleation event (i.e. independent from Eq. (1)). The intersection between both determines the temperature, $T_{\star}=T\left(t_{\star}\right)$, of freezing-relaxation (henceforth homogeneous freezing temperature, HFT), and also fixes $n_{\star}$ and $r_{\star}$. In the literature, freezing temperatures are often vaguely characterised in terms of underlying conditions.

Figure 2 shows the results $n_{\mathrm{i}}^{(1)}(T)$ and $n_{\mathrm{i}}^{(2)}(T)$ for an air parcel lifted with selected updraught speeds. The circles mark the solutions $\left\{n_{\star}, T_{\star}\right\}$. With increasing $w$, the nucleation rate solution (1) shifts to lower $T$, as $n_{\mathrm{i}}^{(1)} \propto \tau_{\mathrm{n}} \propto 1 / w$. The increase of the freezingrelaxation solution (2) with $w$ reflects the scaling in Eq. (22).

For any given $w, n_{\star}$ changes only weakly due to the almost vanishing gradient of $n_{\mathrm{i}}^{(2)}(T)$ and the very steep increase of $n_{\mathrm{i}}^{(1)}(T)$ at the intersections characteristic of the homogeneous freezing process. We therefore expect $n_{\star}$ to be rather insensitive to variations in LWV and $J$. The solution dependencies on $w$ and on $n_{\mathrm{d}}$ and $r_{\mathrm{d}}$ are discussed further in section 4 , which also discusses uncertainties due to processes which are not included in our model. The validity of our direct predictions and inferences is checked in section 5 .

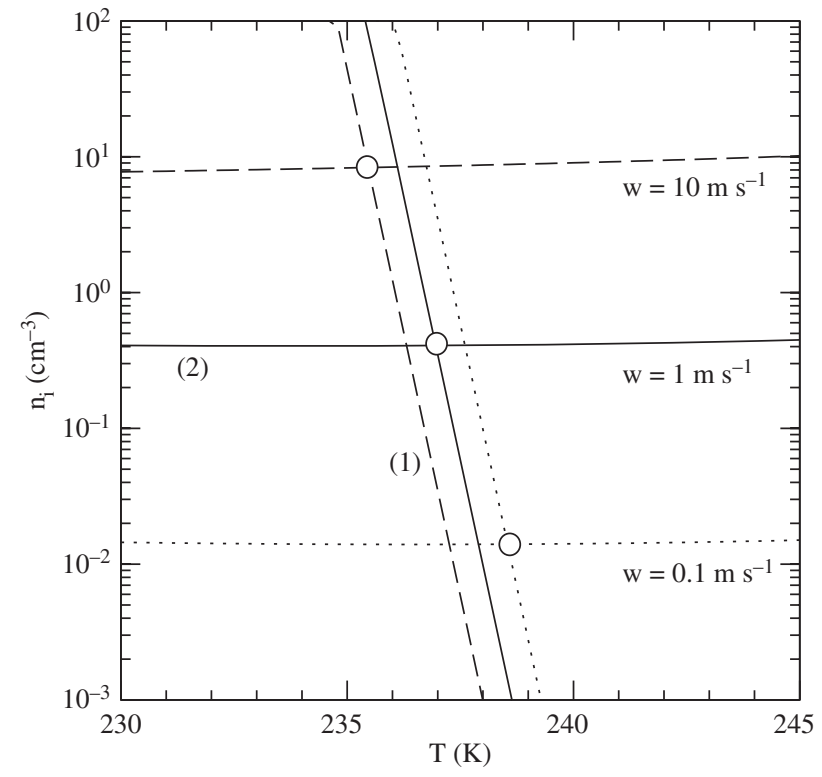

Figure 2. Ice crystal number concentrations from the nucleation model ( 1 , steep curves) and from the freezing-relaxation model (2, flat curves) versus temperature for selected updraught speeds (different line styles). Conditions used in the calculations are $n_{\mathrm{d}}=100 \mathrm{~cm}^{-3}$ and $r_{d}=5 \mu \mathrm{m}$. Intersections circles locate the set of values $\left\{n_{\star}, T_{\star}\right\}$, where the homogeneously frozen droplets begin to quench the supersaturation.

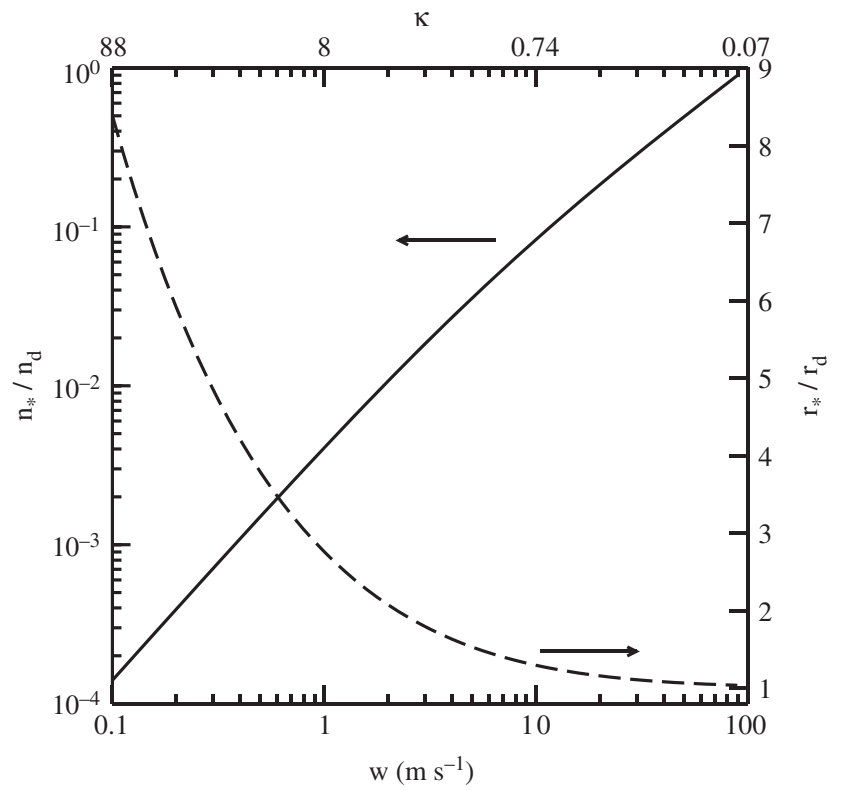

Figure 3. Frozen droplet fractions (solid curve) and mean ice crystal radii relative to initial value (dashed curve) at freezing-relaxation from homogeneous ice nucleation versus updraught speed. Droplet parameters are as in Figure 2.

\section{Results and discussion}

The number of crystals formed at freezing-relaxation in the fastgrowth (low-updraught) regime scales strongly with $w, n_{\star} \propto w^{3 / 2}$. The mean ice crystal radius scales according to $r_{\star} \propto w^{-1 / 2}$, so that the IWC $\propto n_{\star} r_{\star}^{3}$ does not depend on $w$. By contrast, in the slowgrowth (high-updraught) regime, ice properties are unaffected by ice microphysics, since $n_{\star} \propto w$ and $\left\{n_{\star} \rightarrow n_{\mathrm{d}} ; r_{\star} \rightarrow r_{\mathrm{d}}\right\}$. This means that ice crystals nucleating in vigorous deep convective cores initially remove water vapour very quickly at the rate at which the water molecules diffuse to the freezing droplets $\left(\mathcal{L} \simeq 4 \pi D s_{\mathrm{wi}} n_{\mathrm{d}} r_{\mathrm{d}}\right)$ and IWC $\propto w$.

Solutions $n_{\star} / n_{\mathrm{d}}$ (frozen droplet fraction) and $r_{\star} / r_{\mathrm{d}}$ are shown versus $w$ and corresponding $\kappa$ values in Figure 3. The ice crystal number concentration increases with $w$ according to the scaling laws discussed above. The solutions transition from $w^{3 / 2}$-scaling 


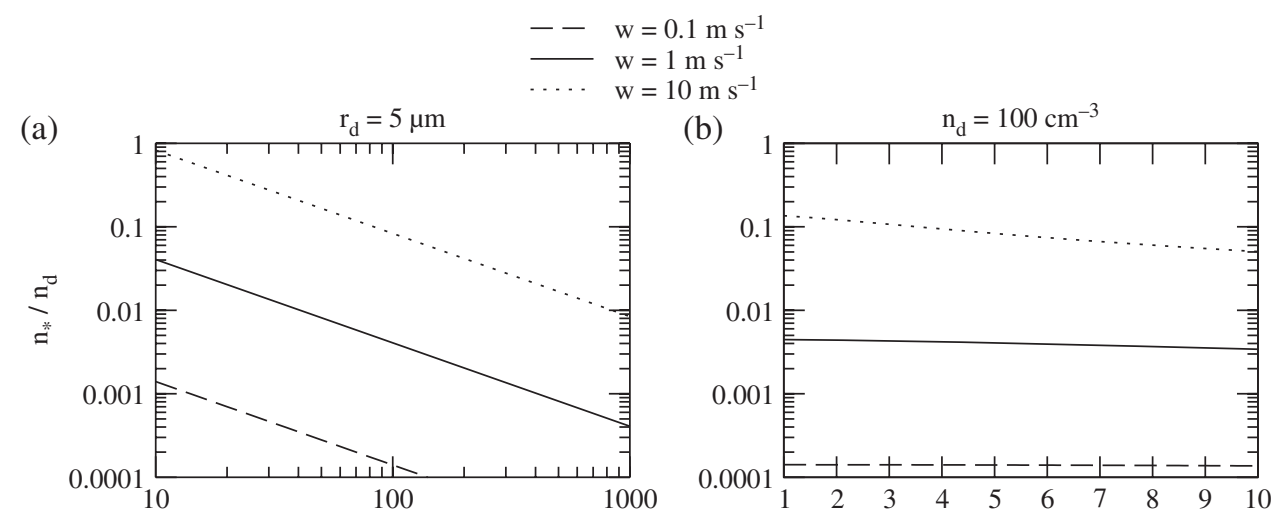

(c)

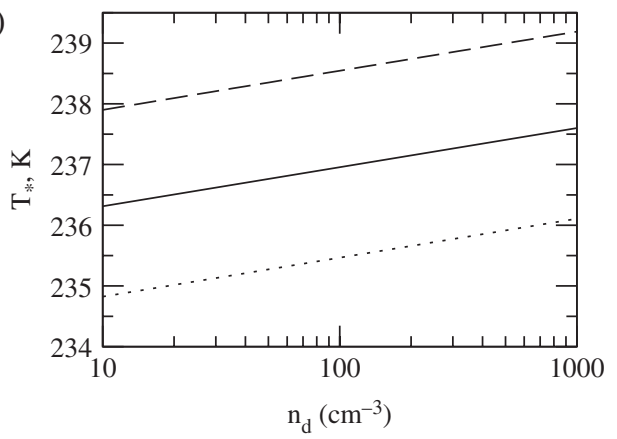

(d)

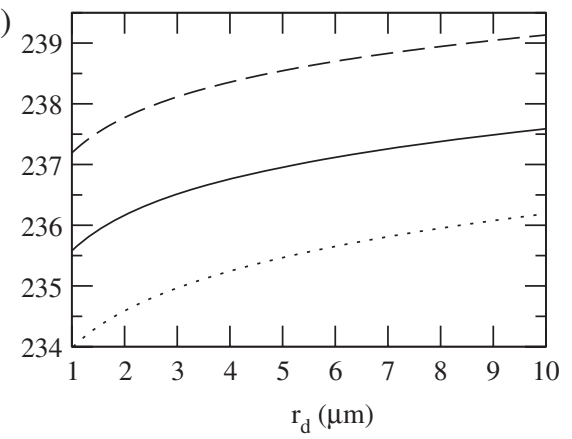

Figure 4. (a,b) Frozen droplet fractions and (c,d) homogeneous freezing temperatures versus $(\mathrm{a}, \mathrm{c})$ total droplet number concentration with $r_{\mathrm{d}}=5 \mu \mathrm{m}$ and $(\mathrm{b}, \mathrm{d})$ mean droplet radii with $n_{\mathrm{d}}=100 \mathrm{~cm}^{-3}$, for different updraught speeds (legend). Compared to numerical simulation results, frozen fractions are overestimated and freezing temperatures are slightly underestimated.

to $w$-scaling above $10-30 \mathrm{~m} \mathrm{~s}^{-1}$, i.e. the linear scaling is realised only for air parcels lifted most vigorously. Vertical wind speeds in deep convective clouds or in strong lee waves may be characterized by $w>1 \mathrm{~m} \mathrm{~s}^{-1}$ (section 6.3).

Taken together, narrow unimodal distributions of copious small ice crystals are expected to be generated in vigorous convective updraughts. In those cases, most of the cloud droplets freeze together rapidly and the ice mode does not grow significantly past the mean droplet size at freezing-relaxation. For $w<10-30 \mathrm{~m} \mathrm{~s}^{-1}$, only a fraction of the cloud droplets freeze, ice crystals grow to large sizes and their size distributions tend to broaden.

Figure 4 provides an overview of the freezing-relaxation solution over a wide range of droplet parameters, $\left\{n_{\mathrm{d}}, r_{\mathrm{d}}\right\}$, and for selected $w$ values. Once again, the dynamical regime dependence of the solutions is manifest in the dramatic dependence of the frozen fraction with $w$ and in significant variations with $w$ of the HFTs, uniquely defined as $T_{\star}=T\left(t_{\star}\right)$. Frozen fractions and HFTs exhibit marked dependencies on $n_{\mathrm{d}}$, when varied over two orders of magnitude (Figure $4(\mathrm{a}, \mathrm{c})$ ). The $n_{\mathrm{d}}$ - and $r_{\mathrm{d}}$-dependence of the frozen droplet fraction (Figure $4(\mathrm{a}, \mathrm{b})$ ) arises from the dependence of the nucleated concentrations on LWV. The $r_{\mathrm{d}}$ dependence becomes significant only for very strong forcing when the supersaturation quenching rate is controlled by $r_{\mathrm{d}}$. The $T_{\star}$-values display a significant $r_{\mathrm{d}}$ dependence (Figure $4(\mathrm{~d})$ ) across the entire range of updraught speeds; this dependence arises from the droplet volume-controlled nature of the homogeneous ice nucleation process.

If ice crystals form in the presence of supercooled water droplets, they grow initially by deposition of water vapour at the expense of evaporating droplets. This process is known as the Wegener-Bergeron-Findeisen (WBF) mechanism occurring for sufficiently large values of $w$ (Korolev and Field, 2008). The WBF is traditionally thought of being triggered by IN at temperatures above the HFT $\left(T>T_{\star}\right)$. Our findings show that this mechanism operates as well in the complete absence of IN in cold cloud outflow regions $\left(T \simeq T_{\star}\right)$.

Finally, we address two issues which are included neither in our theoretical approach nor in the numerical simulations discussed in section 5: the effects on freezing-relaxation of (i) gravitational settling of ice crystals and (ii) pre-existing ice crystals resulting from heterogeneous ice nucleation. They potentially alter nucleated ice crystal number concentrations. Riming and other mechanims relevant for precipitation development are not addressed here.

\subsection{Gravitational settling of ice crystals}

Ice crystals might start to sediment out of the nucleation layer and then no longer contribute to supersaturation quenching. In the case of a sustained updraught, this shifts freezing-relaxation, $t_{\star}$, to a later stage, where more ice crystals nucleate due to colder temperatures. In case of incomplete droplet freezing in weak updraughts, evaporating droplets amplify ice crystal depositional growth, leading to higher fall speeds compared to a high-updraught scenario in which all droplets freeze.

These are counteracting, subtle effects of sedimentation on $n_{\star}$. On the one hand, only few (the largest) ice crystals might be able to settle quickly out of the nucleation layer, reducing $n_{\star}$ only slightly. On the other hand, even small delays of $t_{\star}$ might lead to larger increases in $n_{\star}$ relative to ice crystals lost by sedimentation. Therefore, the true effect of sedimentation depends on the dynamical regime, the depth of the nucleation layer and the vertical fine structure and temporal evolution of supersaturation. The impact of sedimentation on the ice formation process will be larger for lower updraught speeds and for shallower ice supersaturated layers. A characteristic length-scale over which ice forms homogeneously and freezing-relaxation occurs is approximately given by

$$
\Delta z=w \tau_{\mathrm{n}} \simeq \frac{\delta T}{\Gamma_{\mathrm{ws}}} \approx 29 \mathrm{~m},
$$

where we have approximated $K \ll 1$. We can now estimate the ratio between the time-scale of ice crystals to fall out of the nucleation layer, $\tau_{\text {sed }}=\Delta z / v_{\mathrm{t}}$, with the terminal fall speed, $v_{\mathrm{t}}\left(r_{\mathrm{i}}\right)$, and the time-scale of the freezing pulse, $\tau_{\mathrm{n}}$ :

$$
\frac{\tau_{\mathrm{sed}}}{\tau_{\mathrm{n}}}=\frac{w}{v_{\mathrm{t}}} .
$$


Since $v_{\mathrm{t}} \propto r_{\mathrm{i}}^{2}$ for small particles, this ratio scales $\propto w^{2}$ as long as $r_{\mathrm{i}} \simeq r_{\star} \propto w^{-1 / 2}$, and $\propto w$ as $r_{\mathrm{i}} \simeq r_{\star} \rightarrow r_{\mathrm{d}}$. For weak forcing $(w<$ $1 \mathrm{~m} \mathrm{~s}^{-1}$ and $r_{\mathrm{i}}>10 \mu \mathrm{m}$ with $\left.v_{\mathrm{t}}>1 \mathrm{~cm} \mathrm{~s}^{-1}\right)$, we find $\tau_{\text {sed }} / \tau_{\mathrm{n}}<$ 100 , while for strong forcing $\left(w>1 \mathrm{~m} \mathrm{~s}^{-1}\right.$ and $r_{\mathrm{i}}<10 \mu \mathrm{m}$ with $v_{\mathrm{t}}<1 \mathrm{~cm} \mathrm{~s}^{-1}$ ), we find $\tau_{\text {sed }} / \tau_{\mathrm{n}}>100$. Hence, sedimentation may affect freezing-relaxation, but only for weak forcings.

Equation (23) implies that models with vertical grid spacing larger than about $10 \mathrm{~m}$ are incapable of resolving accurately the thin layers over which most ice crystals nucleate homogeneously and will therefore tend to overestimate the frozen droplet fraction. A similar (but relaxed) requirement also holds for IN-induced ice formation (section 6.2).

\subsection{Pre-existing ice crystals}

Let us consider a case where IN do not cause freezing-relaxation before conditions supporting homogeneous freezing are met $\left(T>T_{\star}\right)$. Pre-existing ice crystals deriving from those IN and lofted to higher levels might slow the increase of supersaturation during homogeneous freezing by providing an additional sink for $s_{\mathrm{w}}$. However, diffusional uptake of water vapour is slow due to the small number of ice crystals deriving from IN, so that $s_{\mathrm{w}}$ can hardly be affected. Moreover, ice crystals forming on IN at higher temperatures tend to have large sizes and therefore significant fall speeds, hence they are less likely to be transported to homogeneous freezing levels. Updraughts $>0.2-0.3 \mathrm{~m} \mathrm{~s}^{-1}$ are needed to lift such large ice crystals, but large $w$ values, in turn, favour homogeneous freezing. It is therefore conceivable that effects of pre-existing ice crystals (early freezing IN) on the glaciation of supercooled water layers become important only for low updraught speeds, in which case diabatic processes operating on long time-scales might come into play (Ramaswamy and Detwiler, 1986; Westbrook and Illingworth, 2013).

While we explore the IN issue further in section 6.2, without detailed cloud dynamical simulations the magnitude and importance of effects due to IN and ice crystal settling are difficult to quantify, but it is likely that these effects will be least important in deep convective clouds.

\section{Comparison with numerical simulations}

We have developed a numerical model simulating ice crystal nucleation and depositional growth in moist adiabatic air parcels from a prescribed population of cloud condensation nuclei, the supercooled cloud droplets evolving from them via hygroscopic growth and from various IN types (B. Kärcher, 2016; personal communication). An important feature of it is the non-diffusive treatment of liquid and ice particle growth which we consider essential for an accurate treatment of the homogeneous freezing process. Here we report simulations of homogeneous cloud droplet freezing at constant cooling rates.

For the purpose of comparing directly to our theoretical results, we initialise the cloud parcel model at $T=240 \mathrm{~K}$ $(p \approx 388 \mathrm{hPa})$ and water saturation $\left(s_{\mathrm{w}}=0\right)$, fix $n_{\mathrm{d}}=100 \mathrm{~cm}^{-3}$, assume ice crystals to be spherical and use Eq. (10) for the homogeneous freezing rate coefficient. As the droplets undergo a short condensational growth stage prior to freezing wherein a dynamic equilibrium (quasi-steady state) establishes, we adjust the initial mean droplet radius such that $r_{\mathrm{d}} \approx 5 \mu \mathrm{m}$ is reached around $t_{\star}$.

Figure 5(a) shows the simulated ice crystal number concentrations $\left(n_{\mathrm{i}}\right.$, symbols), either evaluated at $t=t_{\star}$ where the freezing pulse $j$ attains its maximum value $(\star)$, or the total ice numbers evaluated at slightly later times when $j$ decayed to almost zero $(x)$. The former may be compared to the analytical results $\left(n_{\star}\right.$, solid curve), exhibiting similar $w$-scaling but significantly underestimating the simulated values. The agreement in the scaling is encouraging and confirms that the fast-growth (lowupdraught) regime, wherein $n_{\mathrm{i}} \propto w^{3 / 2}$, is realised up to about $5 \mathrm{~m} \mathrm{~s}^{-1}$. Only then does a transition follow toward linear (slow
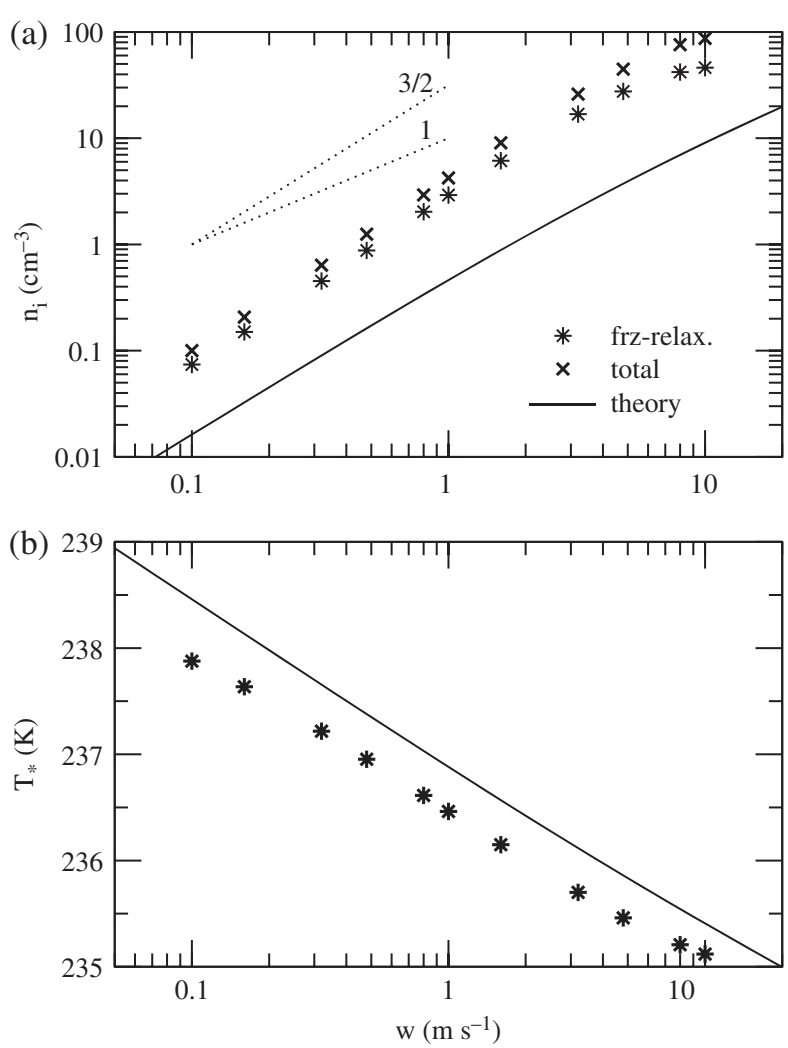

Figure 5. (a) Number concentrations of homogeneously nucleated ice crystals (values either taken at $t=t_{\star}(\star)$ or peak values $(\times)$ attained at later times) versus updraught speed from the numerical simulations (symbols) compared with solutions from the freezing-relaxation theory (solid curve). Dotted lines indicate the theoretical power-law scalings. (b) Simulated homogeneous freezing temperatures $(\star)$, defined at the onset of freezing-relaxation, versus updraught speed compared with solutions from the freezing-relaxation theory (curve).

growth) $w$-scaling. The quantitative discrepancy is most likely tied to the idealised representation of the freezing pulse close to its maximum (Figure 1). Although the simulated pulse is close to exponential for $t<t_{\star}$, this functional form cannot capture the full width of $j$ near $t_{\star}$ and must therefore underestimate ice numbers.

Figure 5(b) shows the same comparison for the HFTs. A logarithmic $w$-scaling is evident in both theory and simulations. The predicted values are slightly higher than the simulated values owing to the underestimation of $n_{\mathrm{i}}$.

We further elaborate the consequences of the step change approximation for $j$ at $t_{\star}$ as displayed in Figure 1. Contrary to this assumption $\left(j\left(t>t_{\star}\right)=0\right)$, ice crystals are still produced at $t>t_{\star}$, amounting to $\Delta n_{\mathrm{i}}=\int_{t_{\star}}^{\infty} j \mathrm{~d} t$ and depending on the width of this portion of the freezing pulse. This width is determined by how quickly the WBF mechanism operates, i.e. how rapidly small yet unfrozen droplets evaporate in the declining supersaturation over water, relative to ongoing freezing. The $n_{\star}$ values taken at freezingrelaxation $(\star)$ are lower than the total nucleated concentrations $(x)$. However, as the numerical results show, those differences are relatively small except for the highest updraught speeds (Figure 5(a)).

To illustrate the solution behaviour in the freezing-relaxation stage, we show in Figure 6(a-i) parcel model results for three selected updraught speeds, either as a function of time elapsed or as a function of vertical distance, $z=w t$, travelled by an air parcel. Figure $6(\mathrm{a}, \mathrm{d}, \mathrm{g})$ depict the temporal evolution of the freezing pulse and the liquid water and ice saturation ratios, (b, e, h) show corresponding altitude profiles of the mean radii and the total number concentrations of droplets and ice crystals and $(c, f, i)$ show the particulate cloud water contents. All simulations are halted at a final temperature of $233 \mathrm{~K}$. (The simulation results shown schematically in Figure 1 represent the case $w=1 \mathrm{~m} \mathrm{~s}^{-1}$.)

The time of freezing-relaxation, corresponding to a vertical displacement $\Delta z_{\star}=w t_{\star}$, is defined as the time (altitude) where 

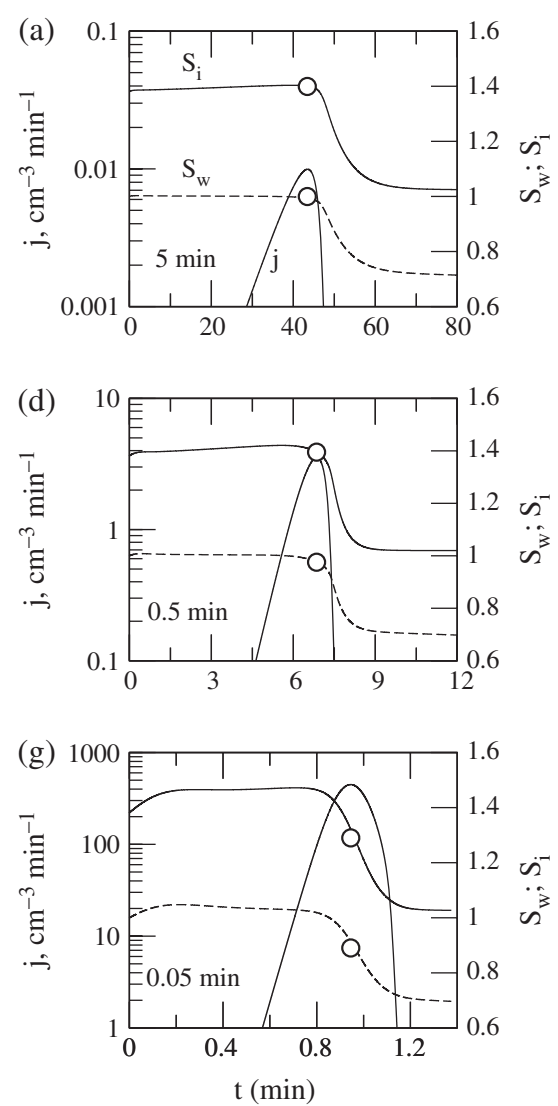
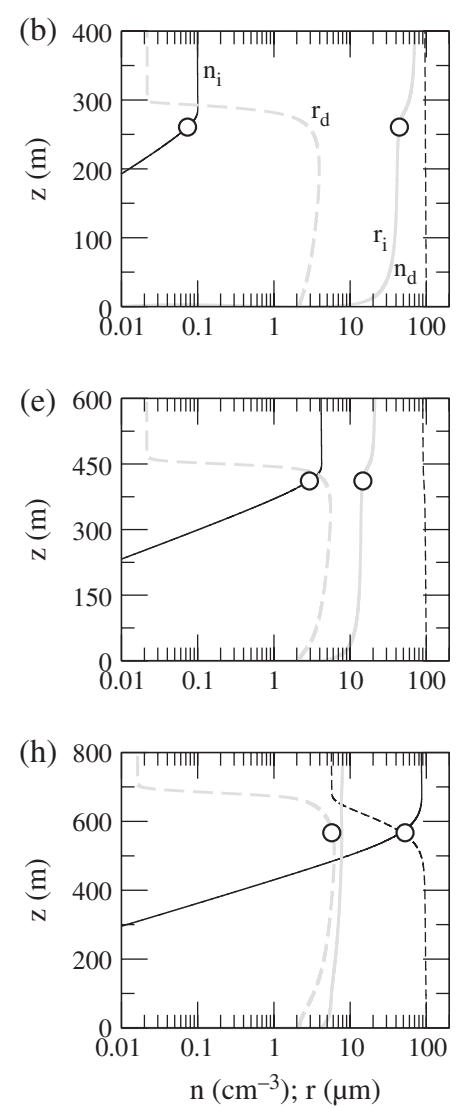
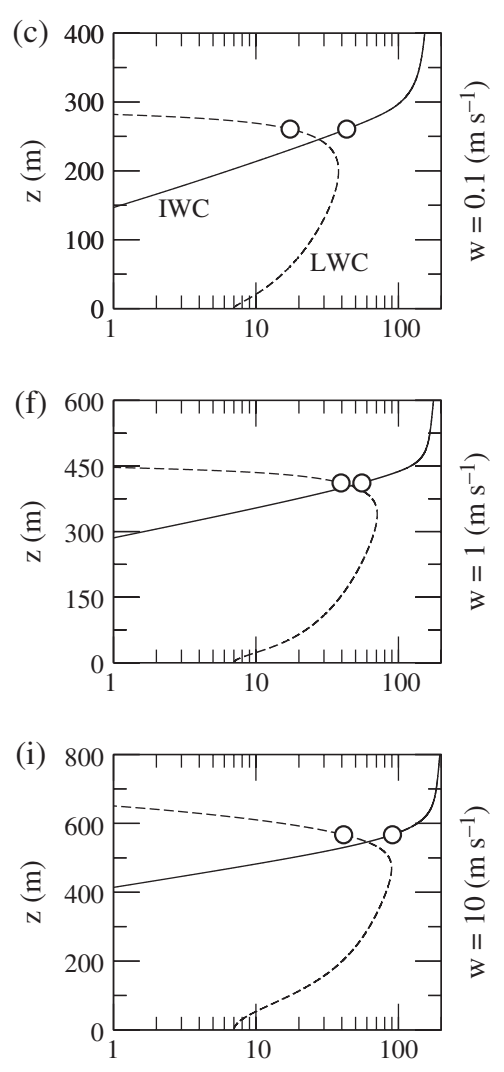

LWC; IWC $\left(\mathrm{mg} \mathrm{m}^{-3}\right)$

Figure 6. Numerical simulations illustrating the homogeneous freezing-relaxation process at three updraught speeds $(\mathrm{a}-\mathrm{c}) w=0.1 \mathrm{~ms} \mathrm{~s}^{-1},(\mathrm{~d}-\mathrm{f}) w=1 \mathrm{~ms} \mathrm{~s}^{-1}$, and $(\mathrm{g}-\mathrm{i}) w=10 \mathrm{~m} \mathrm{~s}^{-1}$. (a, d, g) show the freezing pulse $(j)$ and saturation ratios $\left(S_{\mathrm{w}}, S_{\mathrm{i}}\right) .(\mathrm{b}, \mathrm{e}, \mathrm{h})$ show total number $\left(\right.$ black: $\left.n_{\mathrm{d}}, n_{\mathrm{i}}\right)$ and mean radius $\left(\right.$ grey: $\left.r_{\mathrm{d}}, r_{\mathrm{i}}\right)$ of water particles (dashed curves) and ice crystals (solid). (c, f, i) show liquid water content (LWC, dashed) and ice water content (IWC, solid). (a, d, g) include an estimate of the exponential growth time-scale of the freezing pulse $\left(\tau_{\mathrm{n}} \propto 1 / w\right)$. Open circles mark variables at the simulated time of freezing-relaxation (or equivalently the parcel's corresponding vertical displacement), which are for $w=0.1 \mathrm{~m} \mathrm{~s}^{-1} \sim 44 \min (261 \mathrm{~m})$, for $w=1 \mathrm{~m} \mathrm{~s}^{-1} \sim 7 \mathrm{~min}(411 \mathrm{~m})$, and for $w=10 \mathrm{~m} \mathrm{~s} \sim 1 \mathrm{~min}(567 \mathrm{~m})$.

the freezing pulse reaches its peak (circles) and the water saturation ratios commence to decline. A rapid change of physical variables around this point within a thin vertical layer, consistent with Eq. (23), is indeed seen in Figure 6. In particular, $n_{\mathrm{i}}$ almost reaches peak values, IWC increases strongly and the mean cloud droplet radius $r_{\mathrm{d}}$ (and with it, LWC) diminish dramatically whereby the droplets evaporate almost to their dry aerosol cores. Note that droplet evaporation alone does not reduce $n_{\mathrm{d}}$, only the nucleation of ice acts as a sink to the number of liquid-watercontaining particles.

Comparing the different widths of $j$ at the three updraught speeds explains why the differences between simulated $n_{\mathrm{i}}\left(t_{\star}\right)$ and total $n_{\mathrm{i}}$ values shown in Figure 5 increase with $w$. Whereas the widths generally scale as $\tau_{\mathrm{n}} \propto 1 / w$, as predicted by Eq. (11), they also broaden (in relative terms) with increasing $w$. As a result, the nucleation layer depth in the case of strong convection tends to be larger than predicted by Eq. (23). This happens because the freezing rate coefficient, $J(T)$, increases very rapidly past $t_{\star}$. Therefore, past $t_{\star}$ in strong (weak) updraughts, ice formation is faster (slower) than droplet evaporation. This said, we add that when temperatures become low enough (at the highest updraught speeds in the deepest cloud tops), homogeneous freezing of supercooled aerosol particles entrained from ambient air into the convective cores may add significantly to the ice number budget (Jensen and Ackerman, 2006). This means that $n_{\mathrm{i}}$ values deriving only from cloud droplets are still underestimations in such circumstances.

This discussion suggests that the quantitative theoretical predictions of $\left\{n_{\star}, T_{\star}\right\}$ could be improved by imposing a more suitable functional form of the freezing pulse $j$ around its maximum, where it exerts the largest effect on the resulting integral $\mathcal{R}$ (Eq. (17)) and thereby on $n_{\mathrm{i}}$. A more realistic shape and width of the pulse than provided by Eq. (8) in order to accurately predict total $n_{\mathrm{i}}$ would need to capture the correct $w$ - and $T$-dependencies of the ice formation rate near $t_{\star}$. In any case, freezing at $t>t_{\star}$ must be taken into account.

Furthermore, Figure 6 shows that a substantial amount of liquid water is already evaporated at $t_{\star}$, although $S_{\mathrm{w}}$ is only slightly driven below unity at this instant (except in the case with $w=10 \mathrm{~m} \mathrm{~s}^{-1}$ ), hinting at very rapid droplet evaporation time-scales. Except in vigorous convection, only a small fraction of droplets freeze homogeneously. Only for the strongest forcing (Figure $6(\mathrm{~g}, \mathrm{~h}, \mathrm{i})$ ) does $n_{\mathrm{d}}$ diminish significantly, as most droplets freeze in a burst such that $r_{\mathrm{i}} \simeq r_{\mathrm{d}}$ around $t_{\star}$. Moreover, IWC values after $t_{\star}$ are not very sensitive to $w$. All these features are consistent with our theory and support the freezing-relaxation concept.

We present in Figure 7 droplet and ice crystal size distributions, $n(r)$, extracted from the simulations at freezingrelaxation - defined by $\mathrm{d} j / \mathrm{d} t=0$ during numerical integration consistent with the theory - (a) in a weak stratiform updraught and (b) in a strong deep convective updraught. For slow cooling, small droplets evaporate in the declining supersaturation over liquid water already around $t=t_{\star}$. The ice crystal spectrum is rather broad, and the few nucleated ice crystals have already grown to considerable sizes. For rapid cooling, a higher quasisteady-state liquid water supersaturation establishes prior to freezing-relaxation, leading to slightly larger mean size and narrower size distribution of the droplets at $t_{\star}$. Freezing has already consumed most of the droplets prior to evaporation. Consequently, the ice crystals have not grown much past the mean droplet size and the resulting ice spectrum is quite narrow.

In both cases, ice crystals still nucleate at $t_{\star}$. The two sensitivity studies also demonstrate the low sensitivity to the representation of the droplet spectra, i.e. to variations in LWV and J. As for the latter, we provide a sensitivity simulation using an alternative water activity-based parametrization for J (Koop et al., 2000). All 


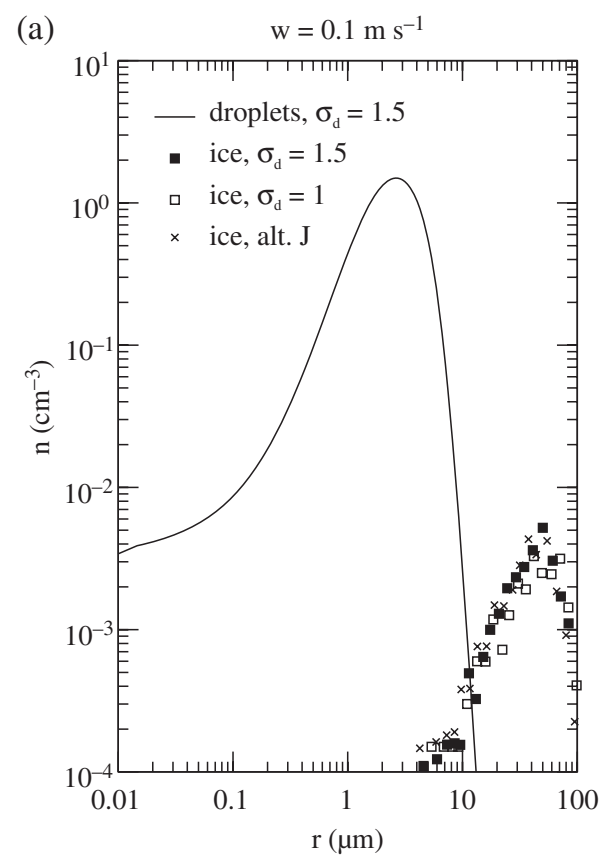

(b)

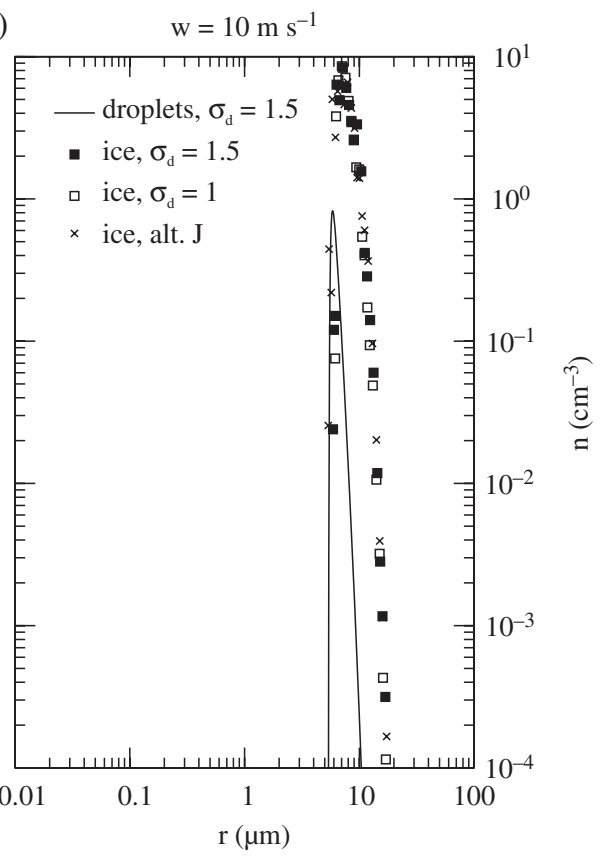

Figure 7. Numerical simulations illustrating droplet (curves) and ice crystal (symbols) size distributions for (a) low and (b) high updraught speeds taken at freezing-relaxation, $t=t_{\star}$. Water droplets (total number concentration $n_{\mathrm{d}}=100 \mathrm{~cm}^{-3}$ ) have been initialised either across a log-normal size distribution with geometric standard deviation $\sigma_{\mathrm{d}}=1.5$ or monodisperse $\left(\sigma_{\mathrm{d}}=1\right.$ ). Filled (open) squares represent ice spectra calculated for a log-normally distributed (monodisperse) droplet population. Crosses represent ice spectra calculated with an alternative homogeneous freezing rate coefficient, $J$.

features discussed by virtue of these results are consistent with our theoretical expectations (sections 2.1 and 3.4).

\section{Atmospheric implications}

We outline ramifications of our model predictions by comparing homogeneous freezing temperatures with field observations (section 6.1) and by deriving estimates of updraught speeds above which homogeneous freezing can be activated in spite of the presence of early freezing IN (section 6.2). These results lend themselves to a discussion of effects of vertical air motion variability (section 6.3).

\subsection{Homogeneous freezing temperatures}

According to laboratory measurements, supercooled pure water droplets with radii $25-50 \mu \mathrm{m}$ freeze homogeneously around $237 \mathrm{~K}$ at cooling rates near $-1 \mathrm{~K} \mathrm{~min}^{-1}$, corresponding to $w \approx 2 \mathrm{~m} \mathrm{~s}^{-1}$ (Riechers et al., 2013). Smaller droplets (radii $1 \mu \mathrm{m}$ ) freeze at lower temperatures, close to $235 \mathrm{~K}$, at similar cooling rates. Homogeneous freezing temperatures in atmospheric clouds depend on LWV, $J$ and the time available for freezing (i.e. on $\tau_{\mathrm{n}}$ ). The latter is constrained by the cooling rate (Eq. (11)) and this dependence is studied in more detailed below.

There is evidence from aircraft measurements for liquid droplets being present in the tops of vigorous convective cells in cumulonimbus clouds at $T=-37.5^{\circ} \mathrm{C}(235.65 \mathrm{~K})$ or perhaps slightly below, with droplets reaching a median volume radius of about $8.5 \mu \mathrm{m}$, droplet number concentrations $150-700 \mathrm{~cm}^{-3}$ and liquid water content $1-2 \mathrm{~g} \mathrm{~m}^{-3}$ (Rosenfeld and Woodley, 2000). An updraught speed of $6 \mathrm{~m} \mathrm{~s}^{-1}$, visually inferred from vertical growth rates of cloud tops, was associated with these measurements. When applying our model at this value of $w$ and $r_{\mathrm{d}}=8.5 \mu \mathrm{m}$ and the observed range of $n_{\mathrm{d}}$, we find $T_{\star}=236.4-236.8 \mathrm{~K}$ which is $\approx 1 \mathrm{~K}$ above the estimated value (measurement uncertainty of perhaps $0.5 \mathrm{~K}$ ). Given the slight overestimation of $T_{\star}$ compared to numerical simulations (Figure 5) and the fact that updraught speeds were not directly measured and could have been slightly higher within the nucleating cells, this suggests that the HFT inferred from the data can be considered to be in line with current understanding of this glaciation mechanism. We add that the rapid decline of liquid water content right below the HFTs seen in the numerical simulations (Figure $6(\mathrm{c}, \mathrm{f}, \mathrm{i})$ ) is also consistent with the field data.

The agreement of predicted $T_{\star}$ with experimental data increases confidence in our physical modelling of homogeneous cloud glaciation processes and the interpretation of how those processes work together. According to Figure 4, HFTs fall below 236-237 K for $w>1 \mathrm{~m} \mathrm{~s}^{-1}$ and increase the lower $w$ and the higher LWV. These results might be useful to check assumptions made in climate models for $T_{\star}$ as a fixed threshold temperature below which supercooled water clouds are assumed to be fully glaciated, irrespective of $w$ and LWV. Recently, Herbert et al. (2015) arrived at a very similar conclusion; we may regard our findings on how low HFTs come about as a theoretical underpinning of their numerical simulation results.

The field observations (Rosenfeld and Woodley, 2000) suggest that homogeneous freezing was the dominant ice nucleation mode and at the same time the presence of IN in the probed clouds was considered plausible. In support of this conjecture, our study suggests that the high updraught speeds provide an explanation for the incapability of IN in controlling droplet freezing processes in the probed convective cloud tops.

We note in passing that the formation of ice crystals in jet aircraft contrails (Kärcher et al., 2015) bears similarity with the ice formation process in deep convective clouds. In jet engine exhaust plumes, copious cloud condensation nuclei are activated into small water droplets that tend to freeze homogeneously in a burst owing to large cooling rates (a few $10 \mathrm{~K} \mathrm{~s}^{-1}$ ). Homogeneous freezing temperatures in contrails are lower than in natural clouds $(229-232 \mathrm{~K})$, due to the significantly shorter time available for nucleation $(\mathcal{O}(0.1 \mathrm{~s}))$ and the much smaller mean droplet sizes (several $100 \mathrm{~nm})$.

\subsection{Activation of homogeneous freezing}

Conceivably, only few of the most efficient IN are left in those air parcels that are exposed to the lowest temperatures (Wiaczek et al., 2010; Westbrook and Illingworth, 2011). IN freezing at higher temperatures should quickly be depleted by precipitation.

According to the freezing-relaxation concept, if the number of early freezing IN is too small to balance the supersaturation 


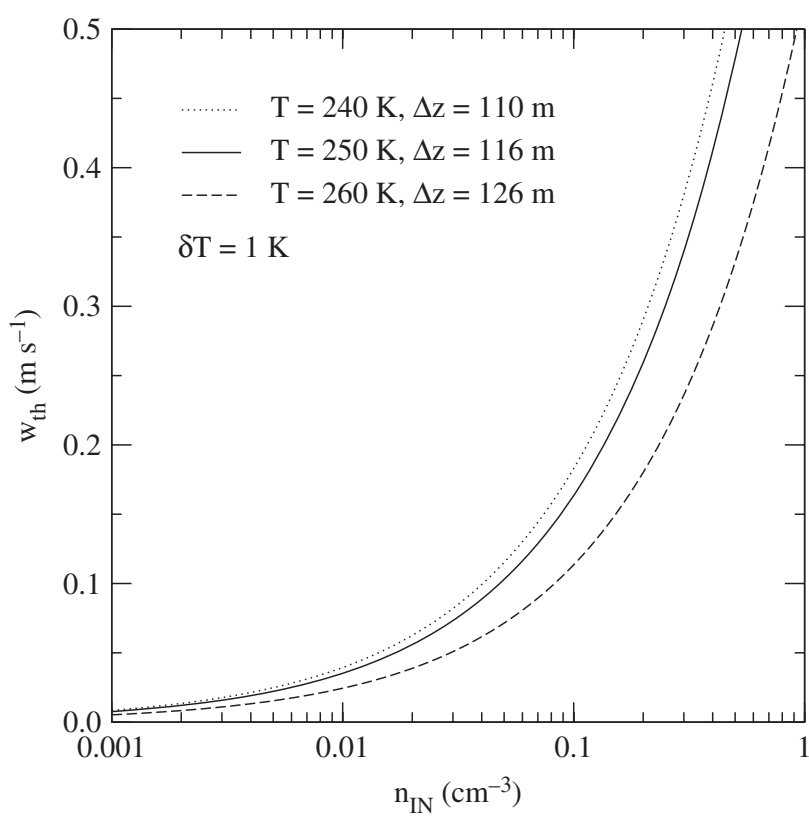

Figure 8. Threshold updraught speeds separating homogeneous and heterogeneous droplet freezing regimes versus ice nuclei number concentration. Values $w>w_{\text {th }}$ activate homogeneous freezing if the air parcel experiences sufficiently low temperatures and have been evaluated at different temperatures for a slope parameter, $\delta T=1 \mathrm{~K}$, of the heterogeneous ice nucleation rate coefficient; $\Delta z$ values denote the resulting depths of the layers in which IN induce ice formation.

forcing for a given updraught speed, then enough liquid droplets may remain in the rising parcel so that homogeneous freezing can subsequently occur. The latter requires further cooling (lifting) after IN activation, in particular for those IN capable of freezing at rather high temperatures (low altitudes). We estimate threshold updraught speeds, $w_{\text {th }}$, separating homogeneous and heterogeneous freezing regimes, by imposing $5 n_{\mathrm{i}}^{(2)} \equiv n_{\mathrm{IN}}$ at $w=w_{\text {th }}$; the factor of 5 is introduced ad hoc in order to account for the underestimation of the ice number in our theoretical model (Figure 5(a)). This leads to

$$
w_{\text {th }}=\left\{\frac{a_{2}}{a_{1}} \frac{\left(2 \pi b_{\mathrm{i}}\right)^{3 / 2}}{v}\left(\frac{\delta T}{\Gamma_{\mathrm{ws}}}\right)^{1 / 2} \frac{n_{\mathrm{IN}}}{5}\right\}^{2 / 3} \propto \Delta z^{1 / 3} n_{\mathrm{IN}}^{2 / 3},
$$

with the freezing-relaxation solution Eq. (22) taken in the limit $\kappa \gg 1$ and the layer depth from Eq. (23). We use the fast growth limit here, because for IN number concentrations $<0.1 \mathrm{~cm}^{-3}$, homogeneous freezing is activated predominantly in the lowupdraught regime; $n_{\text {IN }}$ denotes the total number of IN that can be activated and is not necessarily equal to the total IN number in a sample.

The threshold updraught speed is a nonlinear function of the IN number concentration - reflecting the $w^{3 / 2}$-scaling in the fast-growth regime - and depends rather weakly on temperature, $T$, and nucleation layer depths, $\Delta z=\delta T / \Gamma_{\text {ws }}$. Figure 8 shows $w_{\text {th }}\left(n_{\mathrm{IN}}\right)$ evaluated at temperatures representing potent $(260 \mathrm{~K})$, moderate $(250 \mathrm{~K})$ or poor IN $(240 \mathrm{~K})$ over a range of IN number concentrations as observed in the atmosphere. The temperaturedependence of the heterogeneous ice nucleation rate coefficient is assumed to be flatter than for homogeneous freezing, in line with laboratory studies. For instance, immersion freezing of some type of mineral dust particles may be described by a rate coefficient parametrized with $\delta T=1.14 \mathrm{~K}$ (Herbert et al., 2014), i.e. by a much shallower slope than for homogeneous freezing $(\delta T=0.28 \mathrm{~K})$. Here we use $\delta T=1 \mathrm{~K}$, resulting in significantly thicker layer depths for such heterogeneous nucleation modes, $\mathcal{O}(100 \mathrm{~m})$.

Threshold values for $w$ allowing the activation of homogeneous freezing lie between 10 and $20 \mathrm{~cm} \mathrm{~s}^{-1}$ for upper limit background values of $n_{\mathrm{IN}} \approx 0.1 \mathrm{~cm}^{-3}$. These findings are qualitatively consistent with numerical simulations (Spice et al., 1999), indicating that ice crystal numbers in mixed-phase clouds increase with $w$ and that IN either allow homogeneous freezing to occur if $n_{\mathrm{IN}}$ is low or suppress homogeneous freezing if $n_{\mathrm{IN}}$ is large. Figure 8 also suggests that, for values $w>10 \mathrm{~cm} \mathrm{~s}^{-1}$, most if not all available IN should activate. In other terms - and because the freezing-relaxation concept applies for IN-induced droplet freezing as well - partial activation of IN preferentially occurs in air parcels subject to updraught speeds below about $10 \mathrm{~cm} \mathrm{~s}^{-1}$.

\subsection{Effects of vertical air motion variability}

Our methodology applies to outflow regions of mixed-phase clouds forming during frontal ascent or from cumulus convection. We briefly address the dynamical forcing conditions in a number of tropospheric clouds before discussing effects of small-scale variability in updraught speeds. The latter refers to rapid changes in vertical wind speed and the associated temperature changes encountered by air parcels. Gravity waves are an important mesoscale source of such fluctuations. At the smallest scales, turbulence may add to the resulting forcing of supersaturation by additionally causing humidity fluctuations.

Mean ascent speeds, $w_{0}$, in clouds are variable depending on synoptic conditions. Vertically extended cloud types exposed to low temperatures more likely develop mixed-phase states, because ice nucleation rates increase with decreasing temperature.

At midlatitudes, clouds frequently form in air streams ahead of cold fronts (warm conveyor belts, WCBs). Often originating in the subtropical marine boundary layer, WCBs may reach cirrus levels on the time-scale of a few days crossing a considerable latitude band, resulting in average updraught speeds $w_{0}=0.05-0.1 \mathrm{~m} \mathrm{~s}^{-1}$ averaged over the slantwise ascent (Madonna et al., 2014). Presumably, local $w$ values in upper tropospheric WCB outflow regions, as well as in cloud pockets with embedded convection, are higher. The former are important cirrus cloud source regions and it is conceivable that this (convective) type of cirrus exhibits microphysical properties different from those forming in situ under different dynamical (synoptic) forcing conditions (Jackson et al., 2015).

Among the cloud types of interest here are altocumulus forming in areas of large-scale updraught speeds $w_{0}=0.05-0.1 \mathrm{~m} \mathrm{~s}^{-1}$, and especially cumulus clouds in which convection may cause ascent speeds amounting to $1-5 \mathrm{~m} \mathrm{~s}^{-1}$. Deep convective (cumulonimbus) clouds can develop stronger updraughts in their towering cores, with the most extreme values perhaps occurring in pyro-cumulonimbus originating from wildfires.

We may conclude that mean updraught speeds in the range $0.1-1 \mathrm{~m} \mathrm{~s}^{-1}$ are common values in mid-tropospheric clouds; higher values are associated with deep convective cells. Vertical wind speeds vary around values characterizing the mean ascent. The effect of localised fluctuations of $w$ may be roughly considered by evaluating the freezing-relaxation solution across assumed probability density functions of updraught speeds, $\operatorname{PDF}(w)$. The resulting PDFs of nucleated ice crystal number concentrations depend on the mean updraught speed, $w_{0}$, and an associated scale, $\sigma_{w}$, that serves to indicate the degree of variability around the mean (i.e. the standard deviation in the case of a Gaussian statistic).

To illustrate the effect of $w$ variability on $n_{i}$, in Figure 9 we show PDFs of $n_{*}$ obtained by imposing updraught speeds that are normally distributed around a mean value $w_{0}=0.4 \mathrm{~m} \mathrm{~s}^{-1}$. At the very high Reynolds numbers in mixed-phase clouds, turbulent fluctuations in $w$ are expected to be intermittent, meaning that large fluctuations $\left(w \gg \sigma_{w}\right)$ occur in the distribution wings that are not captured by a Gaussian.

We discuss two cases with weak $\left(\sigma_{w}=0.05 \mathrm{~m} \mathrm{~s}^{-1}\right)$ and stronger $\left(\sigma_{w}=0.15 \mathrm{~m} \mathrm{~s}^{-1}\right)$ fluctuations. In the low- $\sigma_{w}$ case, $\operatorname{PDF}\left(n_{\mathrm{i}}\right)$ already develops a slight skewness towards high $n_{\mathrm{i}}$ values, since $n_{\mathrm{i}}$ increases nonlinearly with $w$ in the given range of vertical wind speeds. A broader distribution with much enhanced skewness develops in the high- $\sigma_{w}$ case. Additional broadening would occur if intermittency was accounted for in $\operatorname{PDF}(w)$. Nonetheless, it is 


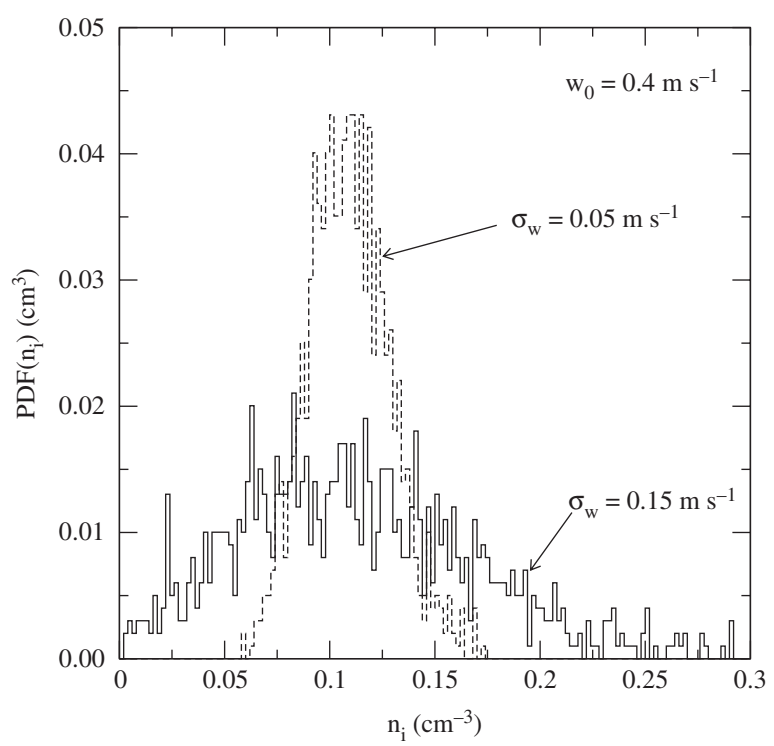

Figure 9. Probability density functions (PDFs) of the number concentrations of ice crystals nucleated homogeneously from cloud droplets. Conditions are as in Figure 2. Updraught speeds are normally distributed around $w_{0}=0.4 \mathrm{~m} \mathrm{~s}^{-1}$, assuming two standard deviations $\sigma_{w}=0.05 \mathrm{~m} \mathrm{~s}^{-1}$ and $\sigma_{w}=0.15 \mathrm{~m} \mathrm{~s}^{-1}$.

evident that vertical air motion variability is capable of causing broad distributions of ice crystal number concentrations (and other ice properties) in mixed-phase clouds, even for rather small vertical velocity variances. This is similar to conditions in cirrus where detailed parcel simulations using $w$ fluctuations lead to broad $\operatorname{PDF}\left(n_{\mathrm{i}}\right)$ consistent with observations (Kärcher and Ström, 2003; Hoyle et al., 2005; Jensen et al., 2013).

This analysis highlights the importance of accurately representing mean vertical velocities and their fluctuations in updraught regions in order to predict primary ice formation in mixed-phase clouds. Global models yield poor estimates of $w_{0}$, especially in convective regions, due to a lack of spatial resolution. Models with kilometre-scale horizontal resolution, including most cloud system-resolving and regional weather prediction models, underestimate $\sigma_{w}$ significantly (Malavelle et al., 2014). These standard deviations were found to be $0.4-0.5 \mathrm{~m} \mathrm{~s}^{-1}$ for shallow low clouds (stratocumulus decks). If they are of similar magnitude in higher-reaching cumulus clouds, then homogeneous freezing may often be activated even if $w_{0}<w_{\text {th }}$, if IN numbers are not unusually high $\left(>0.1 \mathrm{~cm}^{-3}\right)$. This hypothesis is further supported by in situ measurements revealing broad $\operatorname{PDF}(w)$ in midlatitude anvil cirrus comprising a range $\pm 1 \mathrm{~m} \mathrm{~s}^{-1}$ (Muhlbauer et al., 2014) and by numerical analysis of an observed midlatitude cirrus system using large-eddy simulations (Sölch and Kärcher, 2011).

Clearly, this brief discussion of potential effects of vertical air motion variability cannot capture all the processes occurring along real air parcel trajectories for a number of reasons. In particular, the actual $\operatorname{PDF}\left(n_{\mathrm{i}}\right)$ will differ from those presented in Figure 9 since they are based on a model that restricts ice formation processes to first ascent for constant $w$, while in nature:

(i) during freezing-relaxation, the cooling phase may be interrupted by a random warming (due to the stochastic nature of $w$ perturbations), cutting off nucleation events. This means that fewer than the predicted $n_{\star}$ ice crystals are created, most likely in weakly forced events due to the longer duration of the freezing-relaxation pulse and, hence, greater likelihood for such non-persistent freezing events to occur;

(ii) small-scale turbulent mixing and dynamical-microphysical interactions such as latent heating might affect the ice formation process in complex ways;

(iii) changes in $\operatorname{PDF}\left(n_{\mathrm{i}}\right)$ may be brought about by IN altering the supersaturation history, particularly in the case of poor
IN that primarily nucleate near the HFTs. Homogeneous and heterogeneous freezing may occur simultaneously, so that regions within clouds where either nucleation mode occurs will not be temporally and spatially separated;

(iv) sedimentation and turbulent mixing tends to dilute nucleated ice numbers thereby affecting subsequent nucleation events.

Together with the presumably small spatial extent of nucleating cells and the highly transient nature of ice nucleation processes, these issues render a detailed comparison of calculated nucleated ice number with airborne measurements difficult, especially when using fast-moving observational platforms. Some of these issues might be explored with cloud parcel simulations, but only with confidence when the physical characteristics of actual $w$ fluctuations and their dependencies on the large-scale environment are sufficiently well-known.

\section{Conclusions}

\subsection{Summary}

We have studied fundamental processes taking place in the early stage of the evolution of cold mixed-phase stratiform frontal or convective cloud outflow regions forming by homogeneous droplet freezing. The concept of freezing-relaxation underlying our theoretical analysis states that the number of ice crystals is mainly determined by the condition that ice growth-induced water vapour losses reduce the supersaturation at the same rate as it is produced by dynamical forcing due to a prescribed updraught speed. This explains why, contrary to conventional wisdom that heterogeneous IN are required to start the WBF mechanism, this mechanism also occurs in cold supercooled water clouds without such nuclei, leading to small frozen droplet fractions over a wide range of updraught speeds. In cold cloud outflow regions where the homogeneous WBF mechanism operates, fewer and larger ice crystals form leading to larger ice crystal loss rates due to sedimentation in, and presumably to reduced optical depth of, the developing anvil cirrus clouds. Enhanced ice crystal sedimentation may alter the ageing anvil's precipitation efficiency.

An approximate analytical solution of this problem enabled us to study systematically how homogeneously nucleated ice crystal concentrations and associated freezing temperatures vary under changes of updraught speed and cloud droplet number and mean size. We assessed the quantitative accuracy of our results by comparisons with numerical simulations, and found physical dependencies and trends to be well captured. This is encouraging in its own right, as it builds confidence in the current understanding of factors controlling cloud glaciation microphysics. Eliminating quantitative discrepancies in predicted ice number concentrations between theory and simulation might inspire the development of an accurate physically based parametrization scheme. Our results on homogeneous freezing temperatures and inferences regarding IN effects are consistent with numerical studies and observations reported in the literature and are not affected by these discrepancies.

Primary ice crystal number concentrations formed by homogeneous freezing in mixed-phase clouds are to a large extent controlled by the dynamical forcing of supersaturation. The relationship between nucleated concentrations and updraught speed is nonlinear over a wide range of supersaturation forcings. Ever-present small-scale variability in updraught speeds encountered by air parcels results in broad probability distributions of ice crystal number and other mixed-phase cloud properties. We have shown that homogeneous freezing temperatures depend not only on the liquid water content, but also on the forcing, covering a range of values spanning several degrees. We have discussed their dependencies on updraught speed and droplet number and size, and derived estimates for updraught speeds necessary to activate homogeneous freezing in the presence of heterogeneous IN. In conjunction with observational evidence 
of rather large in-cloud vertical air motion variability, this suggests that homogeneous freezing might occur frequently, even in weakly forced mid-level clouds, in the presence of a limited number of heterogeneous IN, often making it the dominant ice formation mechanism in cold cloud outflow regions. If the latter is true, it does not of course rule out that IN might still impact the development of the mixed-phase cloud.

\subsection{Outlook}

Our study suggests a limited role of heterogeneous IN in affecting homogeneous ice formation in high-level supercooled cloud tops. This finding may be particularly important for the characterisation of microphysical properties of the ice phase in deep convective outflow setting the stage for the development of high-altitude anvil clouds. In cold and moist environments with strong vertical wind shear, anvil cirrus originating from mesoscale convective systems tend to be long-lived covering large areas. Widespread anvil cirrus may exert substantial effects on the large-scale circulation due to cloud-radiation feedbacks, but are difficult to represent in global models showing a high sensitivity to the treatment of these clouds. Our findings bear a great potential to assess the ability of models to simulate microphysical and optical properties of fresh anvil cirrus for different large-scale controls They may thereby help to better understand the evolution and radiative response patterns of cold convective detrainment of these upper-level ice clouds in a warmer climate and thus their role in tropical circulation systems, with important implications for hydrological sensitivity and feedbacks on climate.

We expect our findings to be most strongly affected in cases where mean updraught speeds are relatively low, e.g. in midlatitude frontal clouds. If the outflow regions of these clouds do not reach the cold upper troposphere, efficient heterogeneous IN (those active at temperatures above homogeneous freezing levels) are required to induce ice formation. We argue that in such cases frozen IN fractions should likewise display a dependence on the dynamical forcing, but updraught speeds may often be large enough to frequently cause complete activation of all available IN. If the outflow regions of these clouds experience sufficiently cold conditions, then we expect that liquid water layers will be difficult to identify in in situ measurements due to their shallowness. At any rate, knowledge about local updraught speeds is indispensable in predicting the associated ice cloud properties. Accurately capturing vertical air motion variability and ice formation in convective updraught regions in numerical simulations requires a realistic treatment of IN numbers in numerical models with very fine vertical resolution. The latter implies that physical processes governing cloud ice formation need to be parametrized in low-resolution regional and global models.

High-resolution cloud-resolving simulations are necessary to quantify the impact of ice crystal sedimentation and pre-existing ice crystals on homogeneous ice formation. Those simulations should represent all factors controlling spatial and temporal variability in supersaturation, in particular temperature fluctuations and the fine-scale structure of the moisture field. Presumably, those issues tend to be less problematic in deep convective clouds with very large mean updraught speeds. Lofting of liquid or frozen cloud particles and freezing of entrained supercooled aerosol particles are important additional factors controlling the initial microphysical states in the tops of such clouds.

Our main intention here is to spur a scientific dialogue on the relative roles of aerosols and dynamical forcing in atmospheric ice formation. We encourage the development and use of high-resolution simulations (e.g. Dipankar et al., 2015) providing crucial links of supercooled cloud glaciation and cirrus formation processes both to the synoptic confines wherein clouds evolve and the small-scale environment that affects cloud particle formation. In addition to high-resolution dynamical and microphysical modelling using advanced cloud schemes, it would be valuable to test details of atmospheric ice formation processes as described here with suitable field observations. Measurements providing correlations, let alone leading to quantitative relationships, between aerosols, ice cloud variables, large-scale meteorology and small-scale dynamical forcing are very sparse. We therefore hope that our findings facilitate analyses of model simulations and serve as a guide to design and interpret targeted field measurements, especially those capable of providing collocated multi-scale dynamical and microphysical information.

\section{Acknowledgement}

This study emerged within the collaborative research program 'High Definition Clouds and Precipitation for advancing Climate Prediction', $\mathrm{HD}(\mathrm{CP})^{2}$, directed by Bjorn Stevens.

\section{Appendices}

\section{A. Ice supersaturation equation}

Our starting point is the water supersaturation equation derived by Korolev and Mazin (2003), cast into the form

$$
\frac{1}{S_{\mathrm{w}}} \frac{\mathrm{d} S_{\mathrm{w}}}{\mathrm{d} t}=\tilde{a}_{1} w-\tilde{a}_{2} \mathcal{R}
$$

Here, $S_{\mathrm{w}}$ is the water saturation ratio defined as the ratio of the water vapour partial pressure to the water vapour saturation pressure. Throughout our study, saturation vapour pressures over supercooled water and hexagonal ice, as well as latent heats of vaporisation thermodynamically consistent with them, are taken from Murphy and Koop (2005).

We transform Eq. (A1) into Eq. (1) and derive the respective coefficients, $a_{1}$ and $a_{2}$. To this end, we introduce $S_{\text {wi }}$, the ratio of ice and water vapour saturation pressure, yielding the ice saturation ratio, $S_{\mathrm{i}}=S_{\mathrm{wi}} S_{\mathrm{w}}$, and

$$
\frac{\mathrm{d} S_{\mathrm{i}}}{\mathrm{d} t}=\underbrace{S_{\mathrm{i}}\left(\tilde{a}_{1} w+\frac{\mathrm{d} \ln S_{\mathrm{wi}}}{\mathrm{d} T} \frac{\mathrm{d} T}{\mathrm{~d} t}\right)}_{a_{1} w}-\underbrace{\tilde{a}_{2} S_{\mathrm{i}}}_{a_{2}} \mathcal{R} .
$$

The coefficient $a_{2}$ as defined in Eq. (3) follows directly from $\tilde{a}_{2}$ derived by Korolev and Mazin (2003) after a change of variables. To obtain $a_{1}$, we make use of the Clausius-Clapeyron relationship, $\mathrm{d}\left(\ln p_{s}\right) / \mathrm{d}(\ln T)=L /\left(R_{\mathrm{w}} T\right)$, connecting saturation vapour pressures, $p_{s}$, with the respective latent heats of vaporisation, $L$. Differentiating $S_{\mathrm{wi}}(T)$ results in

$$
\frac{\mathrm{d}\left(\ln S_{\mathrm{wi}}\right)}{\mathrm{d} T}=\frac{L_{\mathrm{w}}-L_{\mathrm{i}}}{R_{\mathrm{w}} T^{2}}=-\frac{\theta_{\mathrm{f}}}{T},
$$

describing a supersaturation source tied to the cooling of water droplets at water saturation. Using the original expression $\widetilde{a}_{1}$, introducing the absolute value of the cooling rate, $\vartheta=-\mathrm{d} T / \mathrm{d} t$ and noting that $\theta$ is tied to the updraught speed via the liquid adiabatic lapse rate, $\Gamma_{\mathrm{ws}}$, in the cloudy air parcel prior to freezing (Eq. (5)), we finally obtain $a_{1}$ as defined in Eq. (2). As $\mathrm{d} S_{\mathrm{i}} / \mathrm{d} t=\mathrm{d} s_{\mathrm{i}} / \mathrm{d} t$, this reproduces Eq. (1).

Korolev and Mazin (2003) have studied the characteristic time over which water supersaturation decays (phase relaxation). Ice phase relaxation may be considered as the final stage of the freezing-relaxation process, resulting in substantial depositional growth of the nucleated ice crystals. Both the ice phase relaxation time and the freezing-relaxation process, via $\mathcal{L}$ from Eq. (21), depend on the integral radius, $n_{\mathrm{i}} r_{\mathrm{i}}$. This reinforces the notion that the integral radius of the ice crystal population is a crucial determinant of the microphysics of droplet growth and freezing coupling the moisture field with particle properties, similar to conditions in cold cirrus clouds (Kärcher et al., 2014). 


\section{B. Ice nucleation time-scale}

According to Eqs (7) and (11)

$$
\tau_{\mathrm{n}}^{-1} \equiv \frac{\partial \ln (j)}{\partial t}=\frac{\partial \ln (\mathrm{LWV})}{\partial t}+\frac{\partial \ln (J)}{\partial t} .
$$

The first term in Eq. (B1) consists of two contributions related to the decrease of the droplet number concentration, $n_{\mathrm{d}}$, due to adiabatic change and the increase of the single droplet volume, $V_{\mathrm{d}}=4 \pi r_{\mathrm{d}}^{3} / 3$, due to condensational growth:

$$
\frac{\partial \ln (\mathrm{LWV})}{\partial t}=\frac{\partial \ln \left(n_{\mathrm{d}}\right)}{\partial t}+\frac{\partial \ln \left(V_{\mathrm{d}}\right)}{\partial t} .
$$

We neglect the relatively small adiabatic change in $n_{\mathrm{d}}$ and rewrite

$$
\frac{\partial \ln \left(V_{\mathrm{d}}\right)}{\partial t}=\frac{3}{r_{\mathrm{d}}} \frac{\mathrm{d} r_{\mathrm{d}}}{\mathrm{d} t} \simeq \frac{3 v D n_{\mathrm{ws}}}{r_{\mathrm{d}}^{2}}\left(\mathcal{S}_{\mathrm{w}}-1\right)
$$

where we made use of the diffusional growth law $r_{\mathrm{d}} \mathrm{d} r_{\mathrm{d}} / \mathrm{d} t=$ $v D n_{\mathrm{ws}}\left(\mathcal{S}_{\mathrm{w}}-1\right) /\left(1+B_{\mathrm{w}}\right)$ similar to Eq. (13), assuming $B_{\mathrm{w}} \ll 1$. In order to estimate $\mathrm{d} r_{\mathrm{d}} / \mathrm{d} t$, we need to quantify the small derivations from liquid water saturation in an ascending parcel. The equilibrium saturation ratio prevailing during droplet growth follows from the supersaturation equation for droplets, Eq. (A1), by setting $\mathrm{d} S_{\mathrm{w}} / \mathrm{d} t=0$ :

$$
\mathcal{S}_{\mathrm{w}}=1+\frac{\theta_{\mathrm{w}} \vartheta / T}{4 \pi D n_{\mathrm{d}} r_{\mathrm{d}}},
$$

where we have neglected adiabatic change for consistency and set $S_{\mathrm{w}} \approx 1$ in the forcing term. Equation (B4) depending on the integral droplet radius, $n_{\mathrm{d}} r_{\mathrm{d}}$, is consistent with the quasi-steady state supersaturation derived by Korolev and Mazin (2003) for a two-phase water system composed of water vapour and liquid condensate. In particular, $\mathcal{S}_{\mathrm{w}} \approx 1$ and the associated droplet phase supersaturation relaxation time given by $1 /\left(\theta_{\mathrm{w}} \vartheta / T+4 \pi D n_{\mathrm{d}} r_{\mathrm{d}}\right)$ is very short, on the order of seconds to minutes for typical integral droplet radii, justifying the assumption of an equilibrium droplet growth stage prior to freezing-relaxation in our approach (section 2.2) along with the use of $S_{\mathrm{wi}}$ in Eqs (2) and (13).

The second term in Eq. (B1) is rewritten as

$$
\frac{\partial \ln (J)}{\partial t}=\frac{\partial \ln (J)}{\partial T} \frac{\mathrm{d} T}{\mathrm{~d} t}=\frac{\vartheta}{\delta T},
$$

where we have made use of Eq. (10).
Collecting both contributions that are proportional to the cooling rate, the characteristic (inverse) time-scale of homogeneous freezing takes the form

$$
\tau_{\mathrm{n}}^{-1}=\frac{\vartheta}{\delta T}(1+K), \quad K=v n_{\mathrm{ws}} \frac{\theta_{\mathrm{w}}}{\mathrm{LWV}} \frac{\delta T}{T},
$$

where $K$ embodies the droplet growth contribution, $\partial \ln (\mathrm{LWV}) / \partial t$, to $\tau_{\mathrm{n}}$.

\section{Ice supersaturation sink term}

The starting point for the derivation of the ice supersaturation sink term, $\mathcal{L}=a_{2} \mathcal{R}$, is Eq. (17) into which Eqs (8) and (18) are inserted. Transformation of the integration variable $t^{\prime}$ to $x=\left(t-t^{\prime}\right) / \tau_{\mathrm{n}}$ leads to

$$
\mathcal{R}=\frac{4 \pi b r_{\mathrm{d}}}{v} \underbrace{j(t) \tau_{\mathrm{n}}}_{n_{\mathrm{i}}(t)} \underbrace{\int_{0}^{\infty}(1+\kappa x)^{1 / 2} \mathrm{e}^{-x} \mathrm{~d} x}_{\mathcal{G}(\kappa)} .
$$

The integral $\mathcal{G}$ can be solved in closed form:

$$
\mathcal{G}(\kappa)=1+\frac{\sqrt{\pi \kappa}}{2} \underbrace{e^{1 / \kappa} \operatorname{erfc}\left(\frac{1}{\sqrt{\kappa}}\right)}_{\mathcal{F}(\kappa)},
$$

$$
\text { where } \operatorname{erfc}(x)=1-\left(\frac{2}{\sqrt{\pi}}\right) \int_{0}^{x} \exp \left(-z^{2}\right) \mathrm{d} z
$$

is the complementary error function, and $\mathcal{F}(\kappa)$ can be very accurately approximated by rational function expansion (Abramowitz and Stegun, 1972, p.299, section 7.1.26). Its asymptotic limits are given by $\mathcal{F}(\kappa \ll 1) \rightarrow \sqrt{\kappa / \pi}$ and $\mathcal{F}(\kappa \gg$ $1) \rightarrow 1$, in which case $\mathcal{G}$ takes the asymptotic forms

$$
\mathcal{G}(\kappa) \rightarrow\left\{\begin{array}{cl}
1 & \kappa \ll 1, \\
\sqrt{\pi \kappa} / 2 & \kappa \gg 1 .
\end{array}\right.
$$

Note that since $\kappa \propto 1 / w$, we find that $\mathcal{G} \propto 1 / \sqrt{w}$ in the case $\kappa \gg 1$. 


\section{Notation}

Sub- and superscripts

a (dry) air

d water droplet

$\mathrm{f} \quad$ fusion (phase transition)

g depositional growth of ice crystals

i ice crystal, sublimation

m moist air

$\mathrm{n} \quad$ nucleation of ice crystals

s saturated (thermodynamic

equilibrium) value

$\mathrm{t}$ terminal value

th threshold value for

updraught speed, Eq. (25)

$\mathrm{w} \quad$ water vapour, evaporation

(1) homogeneous freezing model, Eq. (12)

(2) freezing-relaxation model, Eq. (22)

$\star \quad$ freezing-relaxation
Acronyms

Units

HFT homogeneous freezing

K temperature, $T_{\star}=T\left(t_{\star}\right)$

IN heterogeneous ice nuclei

IWC ice water content $=4 \pi \rho_{\mathrm{i}} n_{\mathrm{i}} r_{\mathrm{i}}^{3} / 3 \quad \mathrm{~kg} \mathrm{~m}^{-3}$

LWC liquid water content $=\rho_{\mathrm{W}} \mathrm{LWV} \quad \mathrm{kg} \mathrm{m}^{-3}$

LWV liquid water vol. fraction, Eq. (7)

$\operatorname{PDF}(z) \quad$ prob. density func. of variable $z$

WBF Wegener-Bergeron-Findeisen

WCB warm conveyor belt

\begin{tabular}{|c|c|c|c|c|c|}
\hline \multicolumn{2}{|c|}{ Symbols for variables } & \multicolumn{3}{|l|}{ Units } & \multirow{2}{*}{$\begin{array}{l}\text { Units } \\
\mathrm{m}^{-1}\end{array}$} \\
\hline $\begin{array}{l}\gamma \\
\delta T\end{array}$ & $\begin{array}{l}\text { coefficient of adiabatic change, } c_{p} / R_{\mathrm{a}} \\
\text { slope parameter, Eq. }(10)\end{array}$ & $\begin{array}{l}- \\
\mathrm{K}\end{array}$ & $a_{1}$ & $\begin{array}{l}\text { coefficient in ice supersat. } \\
\text { equation, Eq. (2) }\end{array}$ & \\
\hline$\epsilon$ & mass ratio of water and air molecules & - & $a_{2}$ & coefficient in ice supersat. & $\mathrm{m}^{3} \mathrm{~s}^{-1}$ \\
\hline$\theta$ & scaled latent heat coeff. $=L /\left(R_{\mathrm{W}} T\right)$ & - & & equation, Eq. (3) & \\
\hline$\vartheta$ & absol. value of cooling rate, $-\mathrm{d} T / \mathrm{d} t>0$ & $\mathrm{Ks}^{-1}$ & $b$ & coefficient in particle & $\mathrm{m}^{2} \mathrm{~s}^{-1}$ \\
\hline$\kappa$ & dynamical regime parameter, Eq. (16) & - & & diffusion growth rate, Eq. (14) & \\
\hline$\mu$ & water vap. mass mixing ratio $=\epsilon n_{\mathrm{w}} / n_{\mathrm{a}}$ & - & $c_{p}$ & isobaric specific heat capacity & $\mathrm{J} \mathrm{kg}^{-1} \mathrm{~K}^{-1}$ \\
\hline \multirow[t]{2}{*}{$v$} & volume of a water molecule & $\mathrm{m}^{3}$ & & of dry air & \\
\hline & in ice or liquid water & & $g$ & acceleration of gravity & $\mathrm{m} \mathrm{s}^{-2}$ \\
\hline$\varrho$ & mass density & $\mathrm{kg} \mathrm{m}^{-3}$ & $j$ & ice crystal formation rate & $\mathrm{m}^{-3} \mathrm{~s}^{-1}$ \\
\hline$\tau$ & characteristic time-scale & s & & due to nucleation, $\mathrm{d} n_{i} / \mathrm{d} t$ & \\
\hline$\Gamma$ & lapse rate & $\mathrm{Km}^{-1}$ & $k$ & thermal conductivity (dry air) & $\mathrm{J} \mathrm{m}^{-1} \mathrm{~s}^{-1} \mathrm{~K}^{-1}$ \\
\hline$\Delta z$ & freezing-relaxation layer depth, Eq. (23) & $\mathrm{m}$ & $m$ & molecular mass & $\mathrm{kg}$ \\
\hline $\mathcal{G}$ & auxiliary function, Eq. (C2) & - & $n$ & number concentration & $\mathrm{m}^{-3}$ \\
\hline $\mathcal{L}$ & ice supersaturation loss rate, Eq. (21) & $\mathrm{s}^{-1}$ & $p$ & air parcel pressure & $\mathrm{Pa}$ \\
\hline \multirow[t]{2}{*}{$\widetilde{\mathcal{P}}$} & ice supersaturation production rate, & $s^{-1}$ & $r$ & particle (mean) radius & $\mathrm{m}$ \\
\hline & Eq. $(6)$ & & $s$ & supersaturation & - \\
\hline $\mathcal{R}$ & water vapour removal rate, Eq. (C1) & $\mathrm{m}^{-3} \mathrm{~s}^{-1}$ & $t$ & time & s \\
\hline \multirow[t]{10}{*}{$\mathcal{S}$} & quasi-steady-state saturation ratio, & - & $w$ & updraught speed (>0) & $\mathrm{ms}^{-1}$ \\
\hline & Eq. (B4) & & $z$ & vertical air parcel displacement & $\mathrm{m}$ \\
\hline & & & $B$ & latent heating correction & - \\
\hline & & & $D$ & $\begin{array}{l}\text { to particle growth rate } \\
\text { water vapour diffusion } \\
\text { coefficient in air }\end{array}$ & $\mathrm{m}^{2} \mathrm{~s}^{-1}$ \\
\hline & & & $J$ & nucleation rate coefficient & $\mathrm{m}^{-3} \mathrm{~s}^{-1}$ \\
\hline & & & $L$ & latent heat of phase change & $\mathrm{J} \mathrm{kg}^{-1}$ \\
\hline & & & $R$ & specific gas constant & $\mathrm{J} \mathrm{kg}^{-1} \mathrm{~K}^{-1}$ \\
\hline & & & $S$ & $\begin{array}{l}\text { saturation ratio } \\
\text { (fractional relative humidity) }\end{array}$ & - \\
\hline & & & $T$ & air parcel temperature & $\mathrm{K}$ \\
\hline & & & $V$ & single particle volume & $\mathrm{cm}^{3}$ \\
\hline
\end{tabular}

\section{References}

Abramowitz M, Stegun I. 1972. Handbook of Mathematical Functions. Dover Publications: Mineola, NY.

Bony S, Stevens B, Frierson DMW, Jakob C, Kageyama M, Pincus R, Shepherd TG, Sherwood SC, Siebesma AP, Sobel AH, Watanabe M, Webb MJ. 2015. Clouds, circulation and climate sensitivity. Nat. Geosci. 8: 261-268.

Cantrell W, Heymsfield A. 2005. Production of ice in tropospheric clouds review. Bull. Am. Meteorol. Soc. 86: 795-807.
DeMott PJ, Prenni AJ, Liu X, Kreidenweis SM, Petters MD, Twohy CH, Richardson MS, Eidhammer T, Rogers DC. 2010. Predicting global atmospheric ice nuclei distributions and their impacts on climate. Proc. Natl. Acad. Sci. U.S.A. 107: 11217-11222.

Dipankar A, Stevens B, Heinze R, Moseley C, Zängl G, Giorgetta M, Brdar S. 2015. Large-eddy simulation using the general circulation model ICON. J. Adv. Model. Earth Syst. 7: 963-986, doi: 10.1002/2015MS000431.

Ford IJ. 1998. Ice nucleation in jet aircraft exhaust plumes. In Pollution from Aircraft Emissions in the North Atlantic Flight Corridor (POLINAT2), Air Pollution Research Report 68. EUR 18877, Schumann U. (ed.): 269-287. 
Environment and Climate Research Programme, European Commission DG XII: Brussels.

Herbert RJ, Murray BJ, Whale TF, Dobbie SJ, Atkinson JD. 2014. Representing time-dependent freezing behaviour in immersion mode ice nucleation. Atmos. Chem. Phys. 14: 8501-8520.

Herbert RJ, Murray BJ, Dobbie SJ, Koop T. 2015. Sensitivity of liquid clouds to homogenous freezing parameterizations. Geophys. Res. Lett. 42: 1599-1605, doi: 10.1002/2014GL062729.

Hoyle CR, Luo BP, Peter T. 2005. The origin of high ice crystal number densities in cirrus clouds. J. Atmos. Sci. 62: 2568-2579.

Jackson RC, McFarquhar GM, Fridlin AM, Atlas R. 2015. The dependence of cirrus gamma size distributions expressed as volumes in $\mathrm{N}_{0}-\lambda-\mu$ phase space and bulk cloud properties on environmental conditions: Results from the Small Ice Particles in Cirrus Experiment (SPARTICUS). J. Geophys. Res. 120: 10351-10377, doi: 10.1002/2015JD023492.

Jensen EJ, Ackerman AS. 2006. Homogeneous aerosol freezing in the tops of high-altitude tropical cumulonimbus clouds. Geophys. Res. Lett. 33: L08802, doi: 10.1029/2005GL024928.

Jensen EJ, Lawson RP, Bergman JW, Pfister L, Bui TP, Schmitt CG. 2013. Physical processes controlling ice concentrations in synoptically forced, midlatitude cirrus. J. Geophys. Res. 118: 5348-5360, doi: 10.1002/jgrd.50421.

Joos H, Wernli H. 2012. Influence of microphysical processes on the potential vorticity development in a warm conveyor belt: A case-study with the limited-area model COSMO. Q. J. R. Meteorol. Soc. 138: 407-418.

Kärcher B, Lohmann U. 2002. A Parameterization of cirrus cloud formation: Homogeneous freezing of supercooled aerosols. J. Geophys. Res. 107: 4010, doi: 10.1029/2001JD000470.

Kärcher B, Ström J. 2003. The roles of dynamical variability and aerosols in cirrus cloud formation. Atmos. Chem. Phys. 3: 823-838.

Kärcher B, Dörnbrack A, Sölch I. 2014. Supersaturation variability and cirrus ice crystal size distributions. J. Atmos. Sci 71: 2905-2926.

Kärcher B, Burkhardt U, Bier A, Bock L, Ford IJ. 2015. The microphysical pathway to contrail formation. J. Geophys. Res. 120: 7893-7927, doi: 10.1002/ 2015JD023491.

Koop T, Luo B, Tsias A, Peter Th. 2000. Water activity as the determinant for homogeneous ice nucleation in aqueous solutions. Nature 406: 611-614.

Korolev A, Field PR. 2008. The effect of dynamics on mixed-phase clouds: Theoretical considerations. J. Atmos. Sci. 65: 66-86.

Korolev A, Isaac G. 2003. Phase transformation of mixed-phase clouds. Q. J. R. Meteorol. Soc. 129: 19-38.

Korolev A, Mazin I. 2003. Supersaturation of water vapor in clouds. J. Atmos. Sci. 60: 2957-2974.

Madonna E, Wernli H, Joos H, Martius O. 2014. Warm conveyor belts in the ERA-Interim dataset (1979-2010). Part I: Climatology and potential vorticity evolution. J. Clim. 27: 3-26.
Malavelle FF, Haywood JM, Field PR, Hill AA, Abel SJ, Lock AP, Shipway BJ, McBeath K. 2014. A method to represent subgrid-scale updraft velocity in kilometer-scale models: Implication for aerosol activation. J. Geophys. Res. 119: 4149-4173, doi: 10.1002/2013JD021218.

Matsumoto M, Saito S, Ohmine I. 2002. Molecular dynamics simulation of the ice nucleation and growth process leading to water freezing. Nature 416: $409-413$

Mauritsen T, Stevens B. 2015. Missing iris effect as a possible cause of muted hydrological change and high climate sensitvity in models. Nat. Geosci. 8: 346-351, doi: 10.1038/ngeo2414.

Muhlbauer A, Kalesse H, Kollias P. 2014. Vertical velocities and turbulence in midlatitude anvil cirrus: A comparison between in situ aircraft measurements and ground-based Doppler cloud radar retrievals. Geophys. Res. Lett. 41: 7814-7821, doi: 10.1002/2014GL062279.

Murphy DM, Koop T. 2005. Review of the vapour pressures of ice and supercooled water for atmospheric applications. Q. J. R. Meteorol. Soc. 131: 1539-1565.

Pruppacher HR, Klett JD. 1997. Microphysics of Clouds and Precipitation. Kluwer Academic Publishers: Dordrecht, Netherlands.

Ramaswamy V, Detwiler A. 1986. Interdependence of radiation and microphysics in cirrus clouds. J. Atmos. Sci. 43: 2289-2301.

Riechers B, Wittbracht F, Hütten A, Koop T. 2013. The homogeneous ice nucleation rate of water droplets produced in a microfluidic device and the role of temperature uncertainty. Phys. Chem. Chem. Phys. 15: 5873-5887.

Rosenfeld D, Woodley WL. 2003. Deep convective clouds with sustained supercooled liquid water down to $-37.5^{\circ} \mathrm{C}$. Nature 405 : 440-442.

Sölch I, Kärcher B. 2011. Process-oriented large-eddy simulations of a midlatitude cirrus cloud system based on observations. Q. J. R. Meteorol. Soc. 137: 374-393.

Spice A, Johnson DW, Brown PRA, Darlison AG, Saunders CPR. 1999. Primary ice nucleation in orographic cirrus clouds: A numerical simulation of the microphysics. Q. J. R. Meteorol. Soc. 125: 1637-1667.

Stevens B. 2005. Atmospheric moist convection. Annu. Rev. Earth Planet. Sci. 33: 605-643.

Stevens B, Feingold G. 2009. Untangling aerosol effects on clouds and precipitation in a buffered system. Nature 461: 607-613, doi: 10.1038/ nature 08281.

Westbrook CD, Illingworth AJ. 2011. Evidence that ice forms primarily in supercooled liquid clouds at temperatures $>-27^{\circ} \mathrm{C}$. Geophys. Res. Lett. 38: L14808, doi: 10.1029/2011GL048021.

Westbrook CD, Illingworth AJ. 2013. The formation of ice in a long-lived supercooled layer cloud. Q. J. R. Meteorol. Soc. 139: 2209-2221.

Wiaczek A, Peter T, Lohmann U. 2010. The potential influence of Asian and African mineral dust on ice, mixed-phase and liquid water clouds. Atmos. Chem. Phys. 10: 8649-8667. 